\title{
Recent developments on nano-ZnO catalyzed synthesis of bioactive heterocycles
}

\author{
Bubun Banerjee ${ }^{1}[0$
}

Received: 14 October 2017 / Accepted: 9 November 2017 / Published online: 21 November 2017

(C) The Author(s) 2017. This article is an open access publication

\section{Abstract}

Last decade has seen tremendous applications of nano- $\mathrm{ZnO}$ as a mild, cheap, efficient, commercially available, environmentally benign, non-toxic, reusable, heterogeneous catalyst for the various organic transformations. The present review summarizes the applications of nano- $\mathrm{ZnO}$ as an efficient heterogeneous catalyst for the synthesis of diverse biologically relevant heterocycles reported so far.

\section{Graphical abstract}

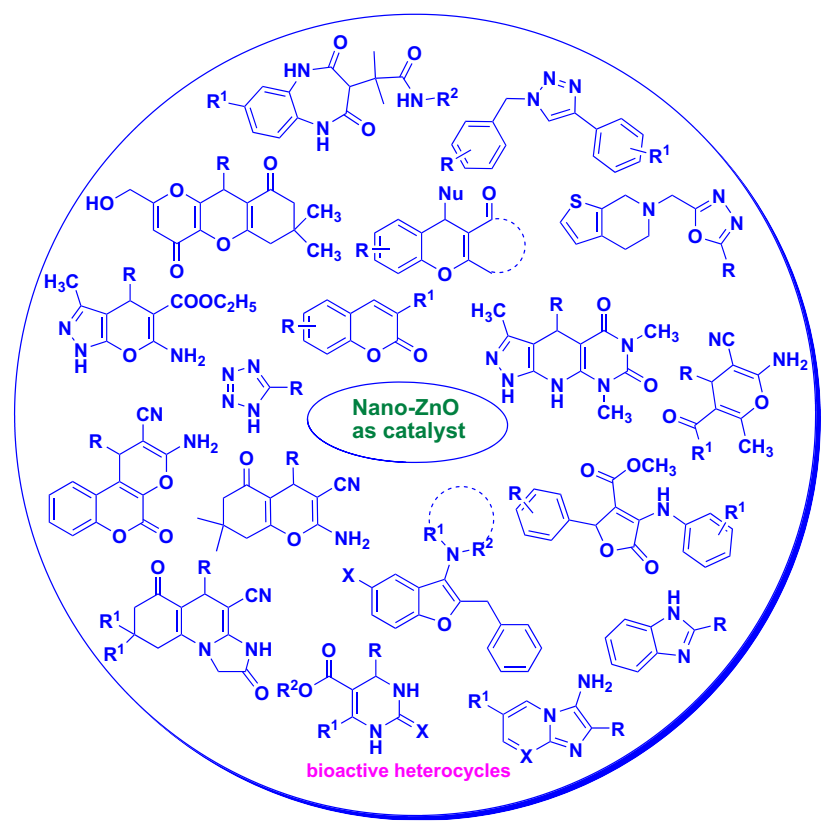

Keywords Bioactive heterocycles $\cdot$ Environmetally benign, Heterogeneous catalysts $\cdot$ Nano-ZnO $\cdot$ Organic synthesis

In the memory of my heavenly grandfather, Amritapada Bandopadhyay.

\section{Bubun Banerjee}

banerjeebubun@gmail.com

1 Department of Chemistry, Indus International University, V.P.O. Bathu, Distt., Una, Himachal Pradesh 174301, India

\section{Introduction}

Heterocycles are the core structural motif in the majority of organic compounds, known so far [1,2]. Heterocyclic moieties are very common in naturally occurring compounds and are important because of their significant biological efficacies that include anticancer [3], cytotoxic [4], anti-malarial [5], anti-microbial [6], anti-inflammatory [7], anti-oxidant 
[8] and many more $[9,10]$. Figure 1 represents a glimpse of marketed drugs containing heterocycles as the core structural unit [11-16].

It is well-established that along with other favorable conditions catalyst plays a crucial role for synthesis of heterocycles $[17,18]$. Worldwide scientists are always trying to modify the catalyst to increase the efficiency of the reaction and to reduce their toxicity level as well. Screening of suitable catalyst is the key to success among the other significant parameters during chemical synthesis. Recently, various nano catalysts have gained much attention due to their greater surface area per unit mass [19]. In recent past, among the other nano catalysts, metal oxides such as $\mathrm{ZnO}, \mathrm{CuO}, \mathrm{SiO}_{2}, \mathrm{CeO}_{2}$, $\mathrm{Fe}_{3} \mathrm{O}_{4}, \mathrm{CaO}, \mathrm{In}_{2} \mathrm{O}_{3} \mathrm{ZrO}_{2}$, etc. in nano form, have drawn considerable attention as efficient, environmentally sustainable, heterogeneous catalysts and have found immense applications in various organic transformations that include $\mathrm{C}-\mathrm{H}$ funtionalizaion [20], synthesis of 2-aminobenzimidazoles,<smiles>COC(=O)C1=C(C)C(=C(C)OC(C)=O)C(C(=O)O[Na])=C(C)N1</smiles>

Nifedipine<smiles>CS(=O)(=O)c1ccc(-c2cn3ccccc3n2)cc1</smiles>

Zolimidine<smiles>O=C1C=C(c2cc(Br)c(O)c(Br)c2)C(=Cc2cc(Br)c(O)c(Br)c2)O1</smiles>

Rubrolide A<smiles>[R]c1ccc(-c2nc3ccc([R])cn3c2CC(=O)N([R])[R])cc1</smiles>

Alpidem; $\mathrm{R}=\mathrm{Cl}, \mathrm{R}^{1}=n \mathrm{Pr}$ Zolpidem; $\mathrm{R}=\mathrm{CH}_{3}, \mathrm{R}^{1}=\mathrm{CH}_{3}$<smiles>COC(=O)C1=C(C)NC(C)=C(C(=O)OC)C1c1cccc2c1OCO2</smiles>

Oxodipine<smiles>Cc1cc2c(s1)Nc1ccccc1N=C2N1CCN(C)CC1</smiles>

olanzapine<smiles>CN1C(=O)CC(=O)N(c2ccccc2)c2cc(Cl)ccc21</smiles>

Clobazam<smiles>CN1CCN(C2=Nc3cc(Cl)ccc3Nc3ccccc32)CC1</smiles>

Clozapine<smiles>CCCSc1ccc2[nH]c(NC(=O)OC)nc2c1</smiles>

Albendazole<smiles>CN=C(NC#N)NCCSCc1nc[nH]c1C</smiles>

Cimetidine<smiles>NNc1nncc2ccccc12</smiles>

Hydralazine<smiles>COc1ccc2[nH]c(S(=O)Cc3ncc(C)c(OC)c3C)nc2c1</smiles>

Omeprazole<smiles>CCOC(=O)c1ncn2c1CN(C)C(=O)c1cc(F)ccc1-2</smiles>

Flumazenil<smiles>CCOC(=O)OCCOC(Cn1cncn1)(Cn1cncn1)c1ccc(F)cc1F</smiles><smiles>CCN(CC)CCCC(C)Nc1ccnc2cc(Cl)ccc12</smiles>

Chloroquine<smiles>Cc1ncc2n1-c1ccc(Cl)cc1C(c1ccccc1F)=NC2</smiles>

Midazolam<smiles>Cc1nccn1CC1CCc2c(c3ccccc3n2C)C1=O</smiles>

Ondansetron<smiles>Cc1ncc([N+](=O)[O-])n1CCO</smiles>

Metronidazole

Fig. 1 Some of the marketed drugs containing heterocycles 
2-aminobenzothiazoles, benzoxazoles [21], tetrahydrobenzofurans [22], $\alpha$-aminophosphonate [23], bis-2,3-dihydroquinazolin-4(1H)-ones [24], 1,4-substituted 1,2,3-triazoles [25], xanthenes [26], pyrano[2,3-d]pyrimidines, $4 H$-chromenes, and dihydropyrano[3,2-c]chromenes [27], substituted pyridines [28], quinoxalin-2-amine [29] and many more [30, 31].

Among these, $\mathrm{ZnO}$-nanoparticles were used in various areas, such as optoelectronics [32,33], ferromagnetism [34], piezoelectric transducers [35], solar cell [36], gas sensors [37], etc. [38]. They also possess antibacterial [39] and antioxidant efficacies [40]. Various $\mathrm{ZnO}$ nanostructures, such as nanoparticles, nanorods, nanowires, nanobelts, nanotubes, nanobridges and nanonails, nanowalls, nanohelixes and polyhedral cages, have been synthesized and well characterized in recent years [41-43]. ZnO-nanoparticles can easily be synthesized from zinc acetate using either sol-gel [44] or precipitation method [45]. In many occasions, it was also synthesized in biogenic pathway using various plant extracts [46]. Thereby, past decade has seen tremendous applications of various morphologies of nano- $\mathrm{ZnO}$ as catalysts in different organic name reactions that include Mannich reaction [47], knoevenagel condensation [48, 49] and in various organic transformations, such as the synthesis of antiplatelet drug (Clopidogrel) [50], phosphonomalonates [51], ferrocenylphosphonates [52], 3-indolyl-3-hydroxy oxindoles [53], $\beta$-acetamido ketones/esters [54], $O$-acylation of alcohol [55], enamination of 1,3-dicarbonyls [56] and $\beta$-amino carbonyl compounds [57], etc.

The favorable physical and chemical properties like mild, low toxicity, low corrosion, large surface area, high pores volume, reusability, low cost, environmental sustainability and commercial availability make this Lewis acidic heterogeneous nano catalyst superior than others.

The present review focuses on the nano- $\mathrm{ZnO}$ catalyzed synthesis of diverse biologically relevant heterocycles and when possible to compare its catalytic efficiency with the rest of the congeners reported so far. The research groups working with this fascinating nano catalyst will surely be attracted by this review.

The following sections describe the catalytic applicability of nano- $\mathrm{ZnO}$ for the synthesis of biologically relevant heterocycles.

\section{Nano-ZnO catalyzed synthesis of $\boldsymbol{N}$-heterocycles}

\section{Synthesis of polysubstituted pyrroles}

Pyrroles are very common in naturally occurring porphyrins, alkaloids and co-enzymes possessing various pharmacological efficacies [58-61]. Some marketed drugs like tallimustine, atorvastatin (Lipitor) contains pyrrol skeleton [62, 63]. Sabbaghan et al. (Table 1) [64] employed ZnO-nanorod as an efficient, reusable catalyst for the synthesis of polysubstituted pyrroles (4) via a one-pot three-component reaction between primary amines (1), dialkyl acetylenedicarboxylates $(\mathbf{2 , 2 a})$ and phenacyl bromide (3) under solvent free conditions at $50{ }^{\circ} \mathrm{C}$. The catalyst was reused for the successive runs without significant loss of its activity. $\mathrm{ZnO}$ nanorod was found to be much more efficient than the commercial $\mathrm{ZnO}$ and even better that the $\mathrm{ZnO}$-nanoparticles or sheets.

\section{Synthesis of imidazoles}

Imidazole and its derivatives possess a wide-range of biological activities that include anti-inflammatory [65], anti-tumor [66], anti-fungal [67] efficacies. Some marketed drugs like omeprazole consist of modified

Table $1 \mathrm{ZnO}$ nanoparticles catalyzed synthesis of polysubstituted pyrroles

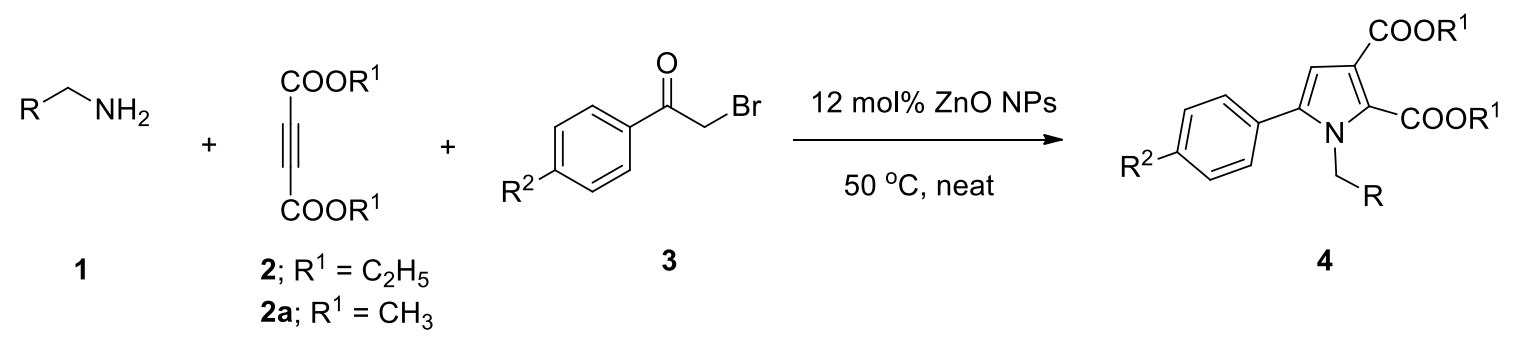

\begin{tabular}{llllllllllll}
\hline Entry & $\mathrm{R}$ & $\mathrm{R}^{1}$ & $\mathrm{R}^{2}$ & Time (min) & Yield (\%) & Entry & $\mathrm{R}$ & $\mathrm{R}^{1}$ & $\mathrm{R}^{2}$ & Time (min) & Yield (\%) \\
\hline 1 & $\mathrm{CH}_{3}$ & $\mathrm{CH}_{3}$ & $\mathrm{H}$ & 45 & 90 & 6 & $4-\mathrm{CH}_{3} \mathrm{C}_{6} \mathrm{H}_{4}$ & $\mathrm{H}$ & $\mathrm{C}_{2} \mathrm{H}_{5}$ & 60 & 86 \\
2 & $\mathrm{CH}_{3}$ & $\mathrm{C}_{2} \mathrm{H}_{5}$ & $\mathrm{H}$ & 45 & 88 & 7 & $4-\mathrm{ClC}_{6} \mathrm{H}_{4}$ & $\mathrm{H}$ & $\mathrm{C}_{2} \mathrm{H}_{5}$ & 60 & 82 \\
3 & $\mathrm{C}_{6} \mathrm{H}_{5}$ & $\mathrm{CH}_{3}$ & $\mathrm{H}$ & 50 & 90 & 8 & $\mathrm{C}_{5} \mathrm{H}_{11}$ & $\mathrm{H}$ & $\mathrm{C}_{2} \mathrm{H}_{5}$ & 45 & 86 \\
4 & $\mathrm{C}_{6} \mathrm{H}_{5}$ & $\mathrm{C}_{2} \mathrm{H}_{5}$ & $\mathrm{H}$ & 50 & 94 & 9 & $\mathrm{CH}_{3}$ & $\mathrm{OCH}_{3}$ & $\mathrm{C}_{2} \mathrm{H}_{5}$ & 60 & 75 \\
5 & $\mathrm{CH}_{3}$ & $\mathrm{CH}_{3}$ & $\mathrm{Cl}$ & 60 & 78 & 10 & $\mathrm{C}_{5} \mathrm{H}_{11}$ & $\mathrm{H}$ & $\mathrm{CH}_{3}$ & 45 & 92 \\
\hline
\end{tabular}


imidazole as a core unit [68]. Nikoofar et al. [69] synthesized $\mathrm{ZnO}$-nanorods. After characterized it by X-ray diffraction (XRD), IR, and scanning electron microscopy (SEM) techniques, they successfully employed this $\mathrm{ZnO}$ nanorods as an efficient, mild, reusable catalyst for the synthesis of 2,4,5-triaryl-1 $\mathrm{H}$-imidazolesin (8) via onepot, three-component reactions of various aldehydes (5), benzils (6) and ammonium acetate (7) in water under reflux conditions (Table 2).

\section{Synthesis of benzimidazoles}

In 2012, Alinezhad et al. (Table 3) [70] reported the synthesis of benzimidazoles (11) from the reaction of $o$-phenylenediamines (9) and formic acid (10) in the presence of nano- $\mathrm{ZnO}$ as catalyst under solvent-free conditions at $70{ }^{\circ} \mathrm{C}$. Very recently, in 2017, Paul et al. [71] have synthesized $\mathrm{ZnO}$-nanoparticles under biogenic pathway from the seeds extract of Parkia roxburghii. Using these $\mathrm{ZnO}$-nanoparticles as catalyst they have synthesized a series of 2-substituted-benzimidazoles (11a) from the reaction of $o$-phenylenediamine (9) and various aldehydes (5) under ultrasonic irradiation in ethanol at room temperature (Table 4).

\section{Synthesis of imidazo-fused polyheterocycles}

Nano-crystalline $\mathrm{ZnO}$ catalyzed simple, efficient, environmentally benign protocol was developed by Swami et al.

Table $2 \mathrm{ZnO}$ nanoparticles catalyzed synthesis of 2,4,5-triaryl- $1 H$-imidazoles<smiles></smiles>

\begin{tabular}{llllllllll}
\hline Entry & $\mathrm{R}$ & $\mathrm{R}^{1}$ & Time $(\mathrm{h})$ & Yield (\%) & Entry & $\mathrm{R}$ & $\mathrm{R}^{1}$ & Time (h) & Yield (\%) \\
\hline 1 & $\mathrm{C}_{6} \mathrm{H}_{5}$ & $\mathrm{H}$ & 2.5 & 80 & 14 & 1-Naphthyl & $\mathrm{H}$ & 3.45 & 78 \\
2 & $4-\mathrm{ClC}_{6} \mathrm{H}_{4}$ & $\mathrm{H}$ & 1.45 & 83 & 15 & 2-furyl & $\mathrm{H}$ & 3.45 & 79 \\
3 & $4-\mathrm{BrC}_{6} \mathrm{H}_{4}$ & $\mathrm{H}$ & 1.45 & 86 & 16 & Cinnamyl & $\mathrm{H}$ & 3.5 & 80 \\
4 & $4-\mathrm{N}\left(\mathrm{CH}_{3}\right)_{2} \mathrm{C}_{6} \mathrm{H}_{4}$ & $\mathrm{H}$ & 3.5 & 75 & 17 & $\mathrm{C}_{6} \mathrm{H}_{5}$ & $\mathrm{Cl}$ & 2.5 & 82 \\
5 & $4-\mathrm{CH}_{3} \mathrm{C}_{6} \mathrm{H}_{4}$ & $\mathrm{H}$ & 3.45 & 81 & 18 & $4-\mathrm{ClC}_{6} \mathrm{H}_{4}$ & $\mathrm{Cl}$ & 2.25 & 87 \\
6 & $4-\mathrm{NO}_{2} \mathrm{C}_{6} \mathrm{H}_{4}$ & $\mathrm{H}$ & 1.15 & 90 & 19 & $4-\mathrm{OCH}_{3} \mathrm{C}_{6} \mathrm{H}_{4}$ & $\mathrm{Cl}$ & 3 & 85 \\
7 & $3-\mathrm{ClC}_{6} \mathrm{H}_{4}$ & $\mathrm{H}$ & 2 & 90 & 20 & $4-\mathrm{CH}_{3} \mathrm{C}_{6} \mathrm{H}_{4}$ & $\mathrm{Cl}$ & 3 & 84 \\
8 & $3-\mathrm{OCH}_{3} \mathrm{C}_{6} \mathrm{H}_{4}$ & $\mathrm{H}$ & 2.25 & 81 & 21 & $4-\mathrm{N}_{2}\left(\mathrm{CH}_{3}\right)_{2} \mathrm{C}_{6} \mathrm{H}_{4}$ & $\mathrm{Cl}_{1}$ & 3.25 & 86 \\
9 & $2-\mathrm{NO}_{2} \mathrm{C}_{6} \mathrm{H}_{4}$ & $\mathrm{H}$ & 1.45 & 78 & 22 & $\mathrm{C}_{6} \mathrm{H}_{5}$ & $\mathrm{CH}_{3}$ & 3.5 & 87 \\
10 & $2-\mathrm{OHC}_{6} \mathrm{H}_{4}$ & $\mathrm{H}$ & 3.25 & 78 & 23 & $4-\mathrm{ClC}_{6} \mathrm{H}_{4}$ & $\mathrm{CH}_{3}$ & 2.45 & 83 \\
11 & $2-\mathrm{OCH}_{3} \mathrm{C}_{6} \mathrm{H}_{4}$ & $\mathrm{H}$ & 3.45 & 70 & 24 & $4-\mathrm{OCH}_{3} \mathrm{C}_{6} \mathrm{H}_{4}$ & $\mathrm{CH}_{3}$ & 4 & 82 \\
12 & $3,5-\left(\mathrm{OCH}_{3}\right)_{2} \mathrm{C}_{6} \mathrm{H}_{3}$ & $\mathrm{H}$ & 4 & 78 & 25 & $4-\mathrm{OCH}_{3} \mathrm{C}_{6} \mathrm{H}_{4}$ & $\mathrm{OCH}_{3}$ & 4 & 80 \\
13 & $2-\mathrm{OH}-6-\mathrm{NO}_{2}-\mathrm{C}_{6} \mathrm{H}_{3}$ & $\mathrm{H}$ & 3.15 & 80 & & & & & \\
\hline
\end{tabular}

Table $3 \mathrm{ZnO}$ nanoparticles catalyzed synthesis of benzimidazoles<smiles>[R]c1ccc(N)c(N)c1</smiles>

9; $1 \mathrm{mmol}$

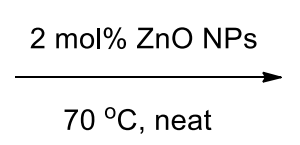

10; $2 \mathrm{mmol}$

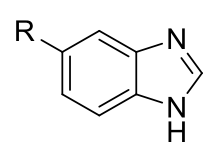

11

\begin{tabular}{llllllll}
\hline Entry & $\mathrm{R}$ & Time $(\mathrm{min})$ & Yield $(\%)$ & Entry & $\mathrm{R}$ & Time $(\mathrm{min})$ & Yield (\%) \\
\hline 1 & $\mathrm{H}$ & 6 & 97 & 4 & $\mathrm{COOH}$ & 120 & 90 \\
2 & $\mathrm{CH}_{3}$ & 30 & 94 & 5 & $\mathrm{COC}_{6} \mathrm{H}_{5}$ & 150 & 92 \\
3 & $\mathrm{OCH}_{3}$ & 240 & 98 & & & & \\
\hline
\end{tabular}


Table $4 \mathrm{ZnO}$ nanoparticles catalyzed synthesis of 2-substituted-benzimidazoles

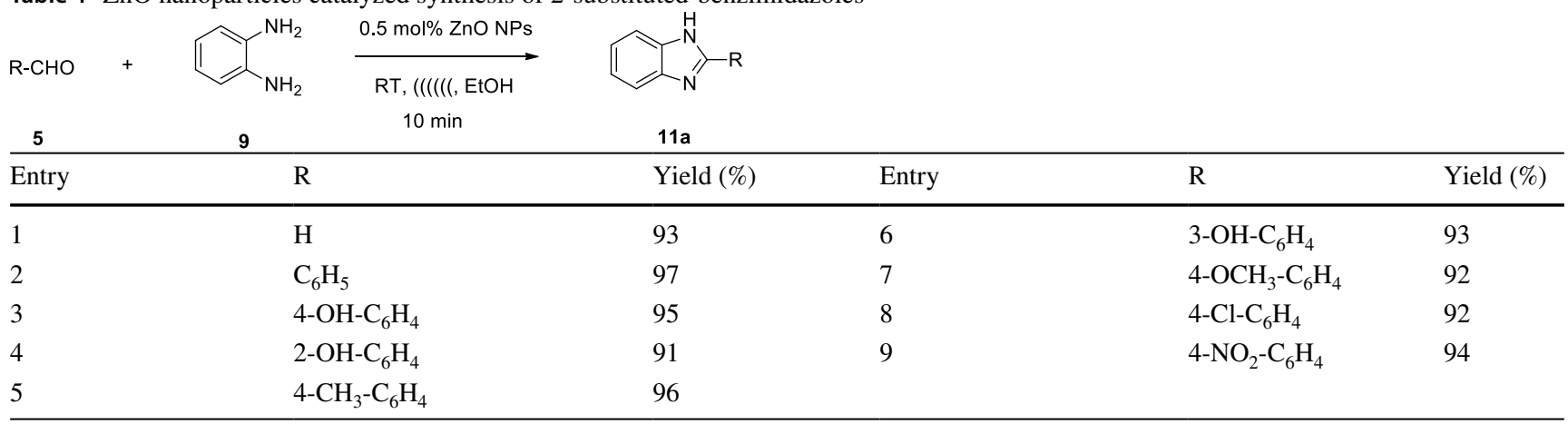<smiles>[R]COC(=O)c1nn(-c2ccc([R])cc2[R])cc1C</smiles>

12

$$
\begin{aligned}
& \mathrm{R}^{1}=\mathrm{Br}, \mathrm{Cl}, \mathrm{H} \\
& \mathrm{R}^{2}=\mathrm{H}, \mathrm{CH}_{3}, \mathrm{Cl}
\end{aligned}
$$<smiles>[R]c1cnc(N)c([R])c1[R]</smiles>

13

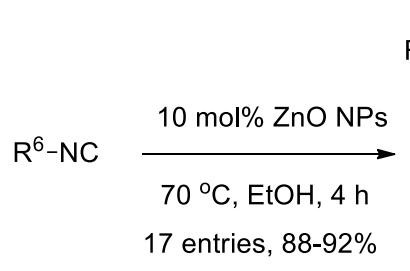

14

$\mathrm{R}^{6}=\mathrm{CH}_{2} \mathrm{COOCH}_{3}, \mathrm{CH}_{2} \mathrm{COOC}_{2} \mathrm{H}_{5}$<smiles>[R7]Nc1c(-c2cn(-c3ccc([R])cc3[R])nc2C(=O)OCC)nc2c([R])c([R])c([R])cn12</smiles>

Scheme $1 \mathrm{ZnO}$ nanoparticles catalyzed synthesis of imidazo-fused polyheterocycles

(Scheme 1) [72] for the synthesis of biologically promising pyrazole coupled imidazo[1,2-a]pyridine derivatives (15) via a one-pot three component reaction between various alkyl-4-formyl-1-phenyl- $1 H$-pyrazole-3-carboxylates (12), 2-aminopyridines (13) and isocyanides (14) in ethanol at $70{ }^{\circ} \mathrm{C}$.

\section{Synthesis of polyhydroquinoline}

A simple, efficient one-pot, four-component condensation of aldehydes (5), dimedone (16), alkyl acetoacetate (17/17a) and ammonium acetate (7) was achieved by Kassaee et al. (Scheme 2) [73] to synthesize 2-methyl-hexahydroquinoline derivatives (19) using $\mathrm{ZnO}$ nano-particles as catalyst under solvent-free conditions at room temperature. Changing alkyl acetoacetate (17/17a) by malononitrile (18) of the four component reaction they also synthesized a series of 2-aminohexahydroquinolines (20) under the same optimized reaction conditions. After completion of the reaction, the catalyst was recovered and reused four times without any significant loss in catalytic activity.

$$
<_{Z}^{\mathrm{CN}}
$$<smiles>[Z]C1=C(N)NC2=C(C(=O)CC(C)(C)C2)C1[R]</smiles>

20

$$
\begin{aligned}
& \text { 18; } Z=C N \\
& 18 a ; Z=\mathrm{COOC}_{2} \mathrm{H}_{5}
\end{aligned}
$$

$10 \mathrm{~mol} \% \mathrm{ZnO}$ NPs

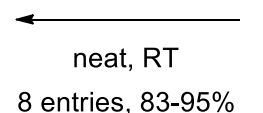

8 entries, $83-95 \%$

7

$\mathrm{R}=\mathrm{C}_{6} \mathrm{H}_{5}, 4-\mathrm{OCH}_{3} \mathrm{C}_{6} \mathrm{H}_{4}, 4-\mathrm{Cl}-\mathrm{C}_{6} \mathrm{H}_{4}, 4-\mathrm{CH}_{3} \mathrm{C}_{6} \mathrm{H}_{4}$<smiles>[R]C(=O)CC(C)=O</smiles>

$$
\text { 17; } \mathrm{R}^{1}=\mathrm{OC}_{2} \mathrm{H}_{5}
$$$$
17 \mathrm{a} ; \mathrm{R}^{1}=\mathrm{OCH}_{3}
$$

$10 \mathrm{~mol} \% \mathrm{ZnO}$ NPs<smiles>[Te][Ba][Ba]</smiles>

26 entries, $84-98 \%$<smiles>[R]C(=O)C1=C(C)NC2=C(C(=O)CC(C)(C)C2)C1[R]</smiles>

19

$\mathrm{R}=\mathrm{C}_{6} \mathrm{H}_{5}, 4-\mathrm{OCH}_{3} \mathrm{C}_{6} \mathrm{H}_{4}, 4-\mathrm{Cl}-\mathrm{C}_{6} \mathrm{H}_{4}, 3-\mathrm{Cl}-\mathrm{C}_{6} \mathrm{H}_{4}, 2-\mathrm{Cl}-\mathrm{C}_{6} \mathrm{H}_{4}$, 4$\mathrm{CH}_{3} \mathrm{C}_{6} \mathrm{H}_{4}, 2,4-$ diClC $_{6} \mathrm{H}_{3}, 3,4-$ diClC $_{6} \mathrm{H}_{3}, 4-\mathrm{Br}-\mathrm{C}_{6} \mathrm{H}_{4}, 4-\mathrm{F}-\mathrm{C}_{6} \mathrm{H}_{4}$, 4- $\mathrm{NO}_{2}-\mathrm{C}_{6} \mathrm{H}_{4}, 3-\mathrm{NO}_{2}-\mathrm{C}_{6} \mathrm{H}_{4}, 2-\mathrm{NO}_{2}-\mathrm{C}_{6} \mathrm{H}_{4}, 4-\mathrm{OH}-\mathrm{C}_{6} \mathrm{H}_{4}$, 4-OH-3$\mathrm{OCH}_{3}-\mathrm{C}_{6} \mathrm{H}_{3}$

Scheme $2 \mathrm{ZnO}$ nanoparticles catalyzed synthesis of polyhydroquinoline 


\section{Synthesis of 1,4-diaryl dihydropyridine derivatives}

$\mathrm{ZnO}$-nanoparticles were found to be an efficient, reusable catalyst for the synthesis of 1,4-diaryl dihydropyridines (21) from the reaction between aldehydes (5), alkyl acetoacetate (17/17a) and substituted anilines (20) (Table 5) [74].

\section{Synthesis of substituted 2,4,6-triaryl pyridines}

Shafiee et al. (Table 6) [75] demonstrated a simple, convenient, $\mathrm{ZnO}$ nanopowder catalyzed condensation between benzaldehydes (5), acetophenones (22) and ammonium acetate (7) for the synthesis of substituted 2,4,6-triaryl pyridines (23) in good yields under solvent-free condition at $120{ }^{\circ} \mathrm{C}$.

\section{Synthesis of quinoxalines}

Sadeghi et al. [76] described $\mathrm{ZnO}$ nanoparticles as an efficient and reusable catalyst for the synthesis of a series of quinoxaline derivatives (24) via the condensation between various 1,2-diketones (6) and 1,2-diamines (9) under solvent-free condition at room temperature (Scheme 3).

Table $5 \mathrm{ZnO}$ nanoparticles catalyzed synthesis of 1,4-diaryl dihydropyridine derivatives<smiles>[R]c1ccc(C=O)cc1</smiles>

5; $1 \mathrm{mmol}$<smiles>[R1]OC(=O)CC(C)=O</smiles>

17/17a; $2 \mathrm{mmol}$<smiles>Nc1c[R]ccc1</smiles>

20; $1 \mathrm{mmol}$

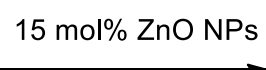

$80^{\circ} \mathrm{C}$, neat<smiles>[R4]CC=C(C)N1C(C)=C(C(=O)O[R12])C(c2ccc([R])cc2)C(C(=O)OCC)=C1C</smiles>

21

\begin{tabular}{llllllllllll}
\hline Entry & $\mathrm{R}$ & $\mathrm{R}^{1}$ & $\mathrm{R}^{2}$ & Time $(\min )$ & Yield (\%) & Entry & $\mathrm{R}$ & $\mathrm{R}^{1}$ & $\mathrm{R}^{2}$ & Time (min) & Yield (\%) \\
\hline 1 & $\mathrm{H}$ & $\mathrm{C}_{2} \mathrm{H}_{5}$ & $\mathrm{H}$ & 120 & 82 & 6 & $\mathrm{H}$ & $\mathrm{C}_{2} \mathrm{H}_{5}$ & $3-\mathrm{CH}_{3}$ & 135 & 85 \\
2 & $\mathrm{H}$ & $\mathrm{C}_{2} \mathrm{H}_{5}$ & $4-\mathrm{Br}$ & 90 & 92 & 7 & $\mathrm{H}$ & $\mathrm{C}_{2} \mathrm{H}_{5}$ & $4-\mathrm{OCH}_{3}$ & 100 & 87 \\
3 & $\mathrm{H}$ & $\mathrm{C}_{2} \mathrm{H}_{5}$ & $4-\mathrm{Cl}$ & 80 & 90 & 8 & $\mathrm{Cl}$ & $\mathrm{C}_{2} \mathrm{H}_{5}$ & $4-\mathrm{OCH}_{3}$ & 120 & 89 \\
4 & $\mathrm{H}$ & $\mathrm{C}_{2} \mathrm{H}_{5}$ & $4-\mathrm{CH}_{3}$ & 110 & 88 & 9 & $\mathrm{Cl}$ & $\mathrm{CH}_{3}$ & $4-\mathrm{CH}_{3}$ & 105 & 88 \\
5 & $\mathrm{H}$ & $\mathrm{C}_{2} \mathrm{H}_{5}$ & $3-\mathrm{Cl}$ & 120 & 86 & 10 & $\mathrm{Cl}$ & $\mathrm{CH}_{3}$ & $3-\mathrm{Br}$ & 109 & 87 \\
\hline
\end{tabular}

Table $6 \mathrm{ZnO}$ nanoparticles catalyzed synthesis of substituted 2,4,6-triaryl pyridines<smiles>[R]c1ccc(C=O)cc1</smiles>

5; $1 \mathrm{mmol}$<smiles></smiles><smiles>CC(=O)ON</smiles>

22; $2 \mathrm{mmol}$

$7 ; 1.3 \mathrm{mmol}$<smiles>[R]c1ccc(-c2cc(-c3cc[R1]([H])cc3)nc(-c3cc[R1](C)cc3)c2)cc1</smiles>

23

\begin{tabular}{lllllllllr}
\hline Entry & $\mathrm{R}$ & $\mathrm{R}^{1}$ & Time (min) & Yield (\%) & Entry & $\mathrm{R}$ & $\mathrm{R}^{1}$ & Time (min) & Yield (\%) \\
\hline 1 & $\mathrm{H}$ & $4-\mathrm{Br}$ & 135 & 86 & 7 & $4-\mathrm{Cl}$ & $4-\mathrm{Cl}$ & 135 & 83 \\
2 & $4-\mathrm{Cl}$ & $4-\mathrm{Br}$ & 120 & 88 & 8 & $\mathrm{H}$ & $4-\mathrm{OCH}_{3}$ & 45 & 75 \\
3 & $4-\mathrm{F}$ & $4-\mathrm{Br}$ & 100 & 91 & 9 & $4-\mathrm{CH}_{3}$ & $4-\mathrm{OCH}_{3}$ & 30 & 82 \\
4 & $4-\mathrm{OCH}_{3}$ & $4-\mathrm{Br}$ & 90 & 95 & 10 & $4-\mathrm{Cl}$ & $4-\mathrm{OCH}_{3}$ & 45 & 78 \\
5 & $\mathrm{H}$ & $4-\mathrm{Cl}$ & 105 & 87 & 11 & $4-\mathrm{NO}_{2}$ & $4-\mathrm{OCH}_{3}$ & 20 & 83 \\
6 & $4-\mathrm{CH}_{3}$ & $4-\mathrm{Cl}$ & 150 & 85 & 12 & $\mathrm{H}$ & $4-\mathrm{OH}$ & 30 & 86 \\
\hline
\end{tabular}




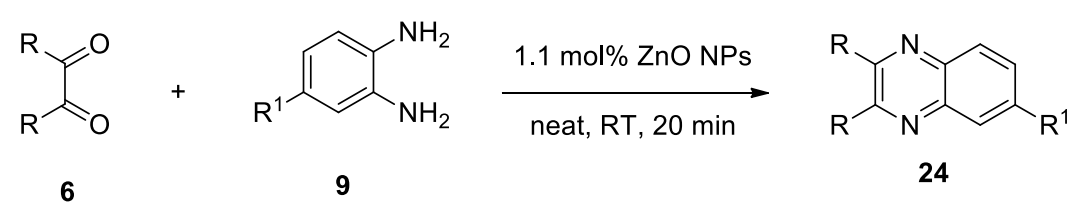<smiles>O=[N+]([O-])c1ccccc1-c1nc2ccccc2nc1-c1ccccc1</smiles>

$92 \%$<smiles>c1cc2c3c(cccc3c1)-c1nc3ccccc3nc1-2</smiles>

$93 \%$<smiles>c1ccc2c3nc4ccccc4c4ccccc4c-3nc2c1</smiles>

$93 \%$<smiles>Cc1ccc2nc(-c3ccccc3)c(-c3ccccc3)nc2c1</smiles>

$96 \%$<smiles>Cc1ccc2nc3c(nc2c1)-c1cccc2cccc-3c12</smiles>

$94 \%$<smiles>Cc1ccc2nc3c4ccccc4c4ccccc4c3nc2c1</smiles>

$95 \%$<smiles>O=[N+]([O-])c1ccc2nc(-c3ccc(F)cc3)c(-c3ccc(F)cc3)nc2c1</smiles><smiles>CCCc1nc2ccccc2nc1C</smiles>

$91 \%$

Scheme $3 \mathrm{ZnO}$ nanoparticles catalyzed synthesis of quinoxalinein

\section{Synthesis of dihydropyrimidinones}

Dihydropyrimidinones (26) possess significant biological efficacies that include antiviral, antibacterial, antihypertensive and antitumor activity [77]. In 1893, Biginelli [78] first reported the synthesis of dihydropyrimidinones
(26) with only 20-50\% yields. In 2015, Hassanpour et al. [79] successfully employed $\mathrm{ZnO}$ nanoparticles as an efficient catalyst for the synthesis of dihydropyrimidinones (26) in good yields via a one-pot three component reaction between various aldehydes (5), alkyl acetoacetate $(\mathbf{1 7 a} / \mathbf{1 7 b})$ and urea (25) or thiourea (25a) in water at

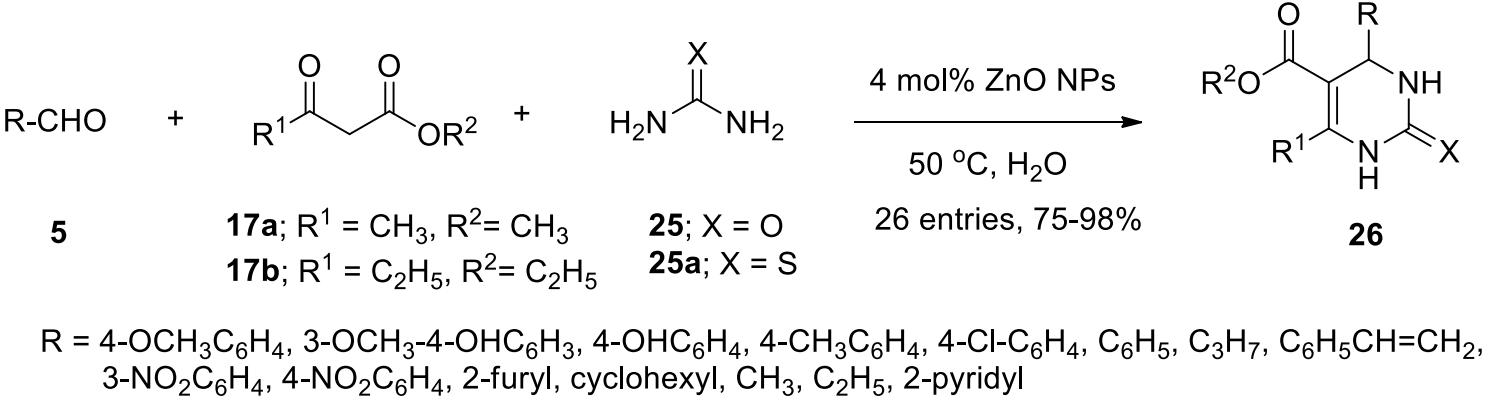

Scheme $4 \mathrm{ZnO}$ nanoparticles catalyzed synthesis of dihydropyrimidinones 
$50{ }^{\circ} \mathrm{C}$. $\mathrm{ZnO}$ nanoparicles was found to be much more efficient than the commercial $\mathrm{ZnO}$ (Scheme 4). After completion of the reaction, the catalyst was recovered and reused successfully without any significant loss in catalytic activity.

\section{Synthesis of hexahydropyrido[2,3-d]pyrimidines}

Abdolmohammadi [80] synthesized a series of hexahydropyrido[2,3- $d$ ]pyrimidine derivatives (29) via the cyclocondensation reaction between arylmethylidenepyruvic acids (27) and 6-aminouracils $(\mathbf{2 8 , 2 8 a})$ in the presence of catalytic amount of $\mathrm{ZnO}$ nanoparticles under solvent-free condition at $70{ }^{\circ} \mathrm{C}$. After completion of the reaction, $\mathrm{ZnO}$ nanoparticles were recovered and recycled three times without any apparent loss in catalytic activity (Table 7).
Synthesis of 1H-pyrazolo[1,2-b] phthalazine-5,10-diones and pyrazolo[1,2-a] [1, 2, 4] triazole-1,3-diones

Azarifar et al. [81] explored the catalytic activity of $\mathrm{ZnO}$ nano-particles for the synthesis of $1 H$-pyrazolo[1,2- $b]$ phthalazine-5,10-diones (32) and pyrazolo $[1,2-a][1,2,4]$ triazole-1,3-dione derivatives (33) via a three-component coupling reaction between aromatic aldehydes $(\mathbf{5})$, malononitrile (18), and phthalhydrazides (30) or 4-arylurazoles (31), respectively, under solvent-free condition at $80-110{ }^{\circ} \mathrm{C}$. High product yields, short reaction times, non-toxicity, easy work-up and reusability of the catalyst are some of the merits of this developed protocol (Scheme 5).

Table $7 \mathrm{ZnO}$ nanoparticles catalyzed synthesis of hexahydropyrido[2,3-d]pyrimidines<smiles>[R]c1ccc(/C=C/C(=O)C(=O)O)cc1</smiles>

27

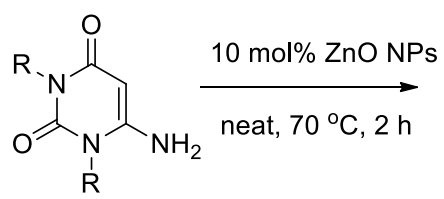

28; $\mathrm{R}=\mathrm{H}$

$28 \mathrm{a} ; \mathrm{R}=\mathrm{CH}_{3}$<smiles>[R]c1ccc(C2C=C(C(=O)O)Nc3c2c(=O)n([R])c(=O)n3[R])cc1</smiles>

29

\begin{tabular}{llllllll}
\hline Entry & $\mathrm{R}$ & $\mathrm{R}^{1}$ & Yield (\%) & Entry & $\mathrm{R}$ & $\mathrm{R}^{1}$ & Yield (\%) \\
\hline 1 & $\mathrm{Cl}$ & $\mathrm{H}$ & 93 & 4 & $\mathrm{Cl}$ & $\mathrm{CH}_{3}$ & 98 \\
2 & $\mathrm{OCH}_{3}$ & $\mathrm{H}$ & 94 & 5 & $\mathrm{OCH}_{3}$ & $\mathrm{CH}_{3}$ & 93 \\
3 & $\mathrm{CH}_{3}$ & $\mathrm{H}$ & 91 & 6 & $\mathrm{CH}_{3}$ & $\mathrm{CH}_{3}$ & 96 \\
\hline
\end{tabular}

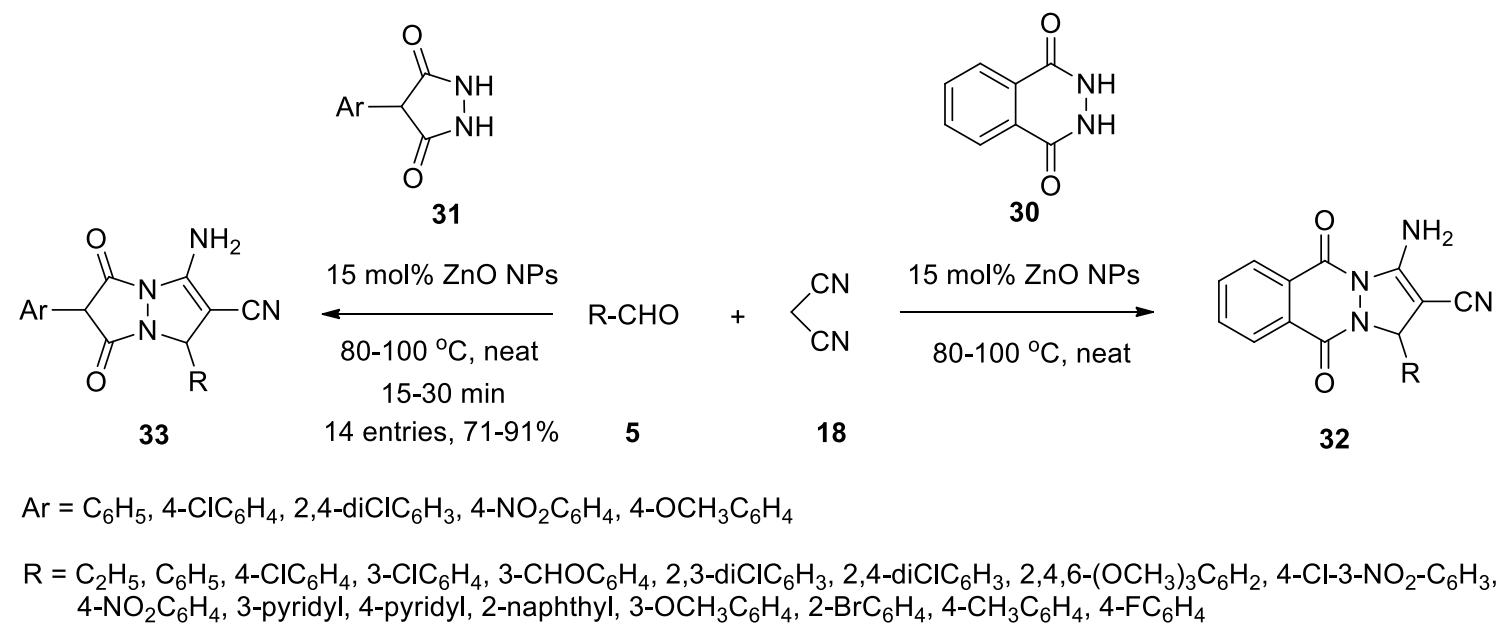

Scheme $5 \mathrm{ZnO}$ nanoparticles catalyzed synthesis of $1 H$-pyrazolo[1,2-b]phthalazine-5,10-diones and pyrazolo[1,2- $a][1,2,4]$ triazole-1,3-diones 
Table $8 \mathrm{ZnO}$ nanoparticles catalyzed synthesis of imidazo[1,2- $a]$ quinoline

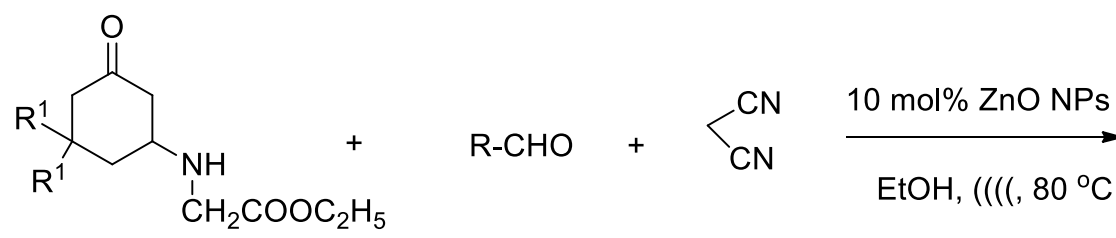

34; $\mathrm{R}^{1}=\mathrm{CH}_{3}$

5

18<smiles>[R]C1C(C#N)=C2NC(=O)CN2C2=C1C(=O)CC([R])([R])C2</smiles>

35

\begin{tabular}{llllllllll}
\hline Entry & $\mathrm{R}$ & $\mathrm{R}^{1}$ & Time (min) & Yield (\%) & Entry & $\mathrm{R}$ & $\mathrm{R}^{1}$ & Time (min) & Yield (\%) \\
\hline 1 & $\mathrm{C}_{6} \mathrm{H}_{5}$ & $\mathrm{CH}_{3}$ & 45 & 90 & 4 & $\mathrm{C}_{6} \mathrm{H}_{5}$ & $\mathrm{H}$ & 48 & 89 \\
2 & $4-\mathrm{CH}_{3} \mathrm{C}_{6} \mathrm{H}_{4}$ & $\mathrm{CH}_{3}$ & 48 & 88 & 5 & $4-\mathrm{CH}_{3} \mathrm{C}_{6} \mathrm{H}_{4}$ & $\mathrm{H}$ & 55 & 88 \\
3 & $4-\mathrm{ClC}_{6} \mathrm{H}_{4}$ & $\mathrm{CH}_{3}$ & 42 & 92 & 6 & $4-\mathrm{ClC}_{6} \mathrm{H}_{4}$ & $\mathrm{H}$ & 45 & 90 \\
\hline
\end{tabular}

\section{Synthesis of imidazo[1,2-a]quinoline}

Imidazo[1,2- $a$ ]quinolines possess immense biological efficacies that include antiallergic [82], anxiolytic [83] activity. Recently, in 2014, a simple, efficient and ultrasound-assisted convenient protocol was developed by Abaszadeh et al. [84] for the synthesis of a series of imidazo[1,2-a]quinoline derivatives (35) via a one-pot three-component reaction between cyclic enaminoketones (34), aromatic aldehydes (5) and malononitrile (18) in the presence of catalytic amount of $\mathrm{ZnO}$ nanoparticles in $\mathrm{EtOH}$ at $80^{\circ} \mathrm{C}$ (Table 8).

\section{Synthesis of imidazo[1,2-a]pyridine/pyrimidine derivatives}

Imidazo[1,2- $a$ ]pyridine/pyrimidines have gained significant attention from the pharmaceutical industry because of their promising biological efficacies that include antibacterial, antifungal, antiviral and anti-inflammatory activities [85, 86]. Many marketed drugs such as alpidem (anxiolytic), zolpidem (hypnotic), and zolimidine (antiulcer) contains imidazo[1,2-a]pyridine as the core structural unit [87, 88]. Sadjadi et al. [89] successfully employed $\mathrm{ZnO}$-nanorods as an efficient, cost effective catalyst for the rapid synthesis of imidazo[1,2- $a$ ]pyrimidines (38) and imidazo[1,2-a]pyridines (39) via the one-pot three-component condensation between benzaldehydes (5), trimethylsilylcyanide (37) and pyrimidin-2-amines (36) or pyridin-2-amines (36a), respectively, in ethanol under the influence of ultrasonic irradiation at room temperature. After completion of reaction they were able to recover $\mathrm{ZnO}$ nanoparticles and reused it up to third run without any significant loss in its catalytic activity (Table 9).

Table $9 \mathrm{ZnO}$ nanoparticles catalyzed synthesis of imidazo[1,2- $a$ ]pyridine/pyrimidines

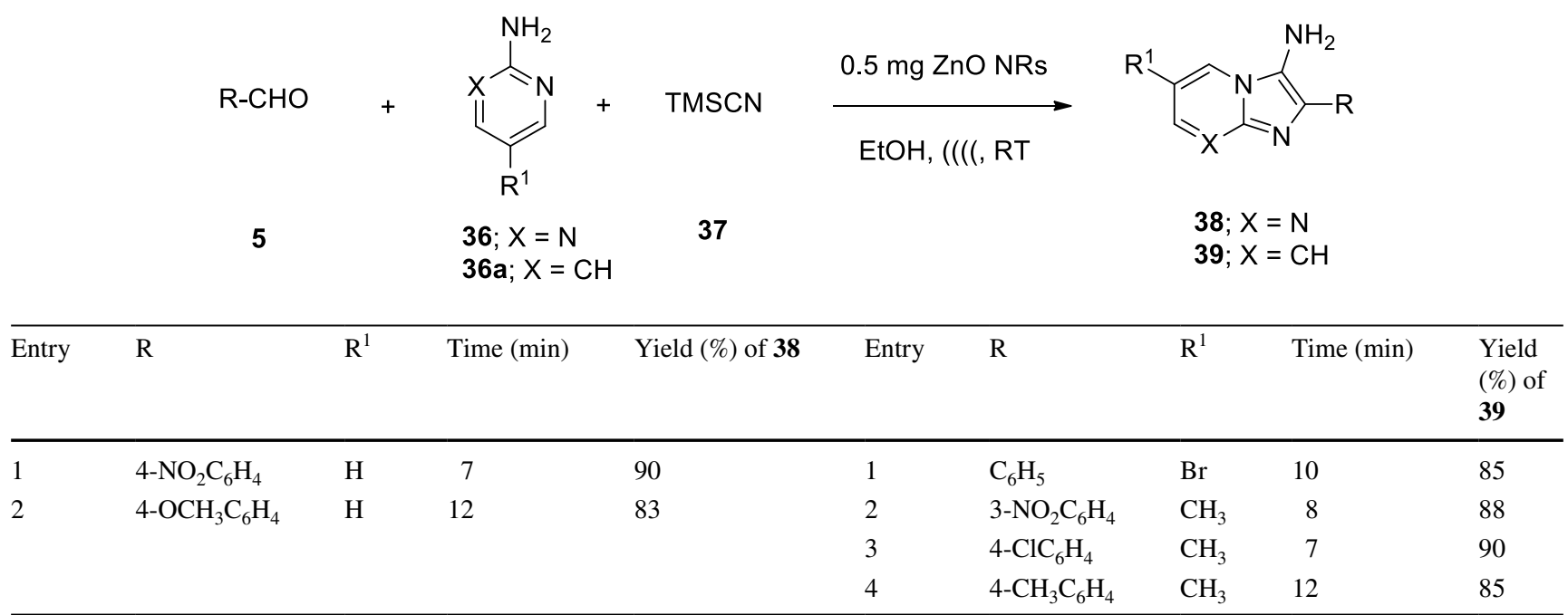




\section{Synthesis of 5-substituted 1H-tetrazoles}

A simple, mild and eco-friendly method was developed for the synthesis of 5-substituted $1 H$-tetrazoles (42) via the cycloaddition reaction of various nitriles $(\mathbf{4 0})$ and sodium azide (41) using $\mathrm{ZnO}$ nanoparticles as a heterogeneous, reusable catalyst in DMF as solvent at $120-130{ }^{\circ} \mathrm{C}$ (Table 10) [90].

\section{Synthesis of benzo[b][1,5]diazepines}

Benzodiazepine represents core structural motif in many marketed drugs such as olanzapine and clozapine (schizophrenia treatment) [91], clobazam (anxiolytic agents) [92], etc. Ghasemzadeh et al. (Table 11) [93] reported a mild, simple and convenient approach for the efficient synthesis of a series of biologically promising benzo[b][1,5]diazepines (44) via one-pot three-component reactions of aromatic diamines (9), various isocyanides (14) and Meldrum's acid (43) in the presence of a catalytic amount of $\mathrm{ZnO}$ nanoparticles in dichloromethane at room temperature. For this transformation, $\mathrm{ZnO}$ nanoparicles were found to be much more efficient than the commercial $\mathrm{ZnO}$. After completion of the reaction, they were able to recover $\mathrm{ZnO}$ nanoparticles and reused six times without apparent loss in catalytic activity.

\section{Nano-ZnO catalyzed synthesis of O-heterocycles}

\section{Synthesis of furan derivatives}

Furans are very common in naturally occurring bioactive heterocycles. This important structural motif has gained considerable attention because of its significant biological efficacies. Many marketed drugs such as rubrolide, sarcophine, benfurodil hemisuccinate $[94,95]$ contain furan skeleton. $5 H$-Furan-2-one derivatives exhibit many pharmacological and biological activities including antifungal, antibacterial, anti-oxidants, anti-inflammatory, anti-microbial and anticancer agents [96-100]. Benzo[b]furan containing heterocycles possesses immense pharmaceutical efficacies that include antifungal [101], antitumor [102] activity. Tekale et al. [103] synthesized a series of biologically promising 3,4,5-trisubstituted furan-2 $(5 H)$-one derivatives $(\mathbf{4 5})$ by the one-pot three-component condensation between various

Table $10 \mathrm{ZnO}$ nanoparticles catalyzed synthesis of 5-substituted $1 H$-tetrazoles

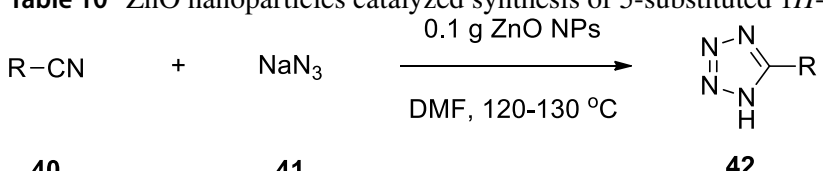

40

41

42

\begin{tabular}{llllllll}
\hline Entry & $\mathrm{R}$ & Time $(\mathrm{min})$ & Yield $(\%)$ & Entry & $\mathrm{R}$ & Time $(\mathrm{min})$ & Yield (\%) \\
\hline 1 & $\mathrm{C}_{6} \mathrm{H}_{5}$ & 14 & 72 & 5 & $4-\mathrm{CHOC}_{6} \mathrm{H}_{4}$ & 14 & 69 \\
2 & $4-\mathrm{ClC}_{6} \mathrm{H}_{4}$ & 14 & 74 & 6 & $4-\mathrm{ClC}_{6} \mathrm{H}_{4} \mathrm{CH}_{2}$ & 24 & 71 \\
3 & $2-\mathrm{ClC}_{6} \mathrm{H}_{4}$ & 14 & 70 & 7 & 2 -pyridyl & 6 & 79 \\
4 & $2-\mathrm{CNC}_{6} \mathrm{H}_{4}$ & 24 & 81 & 8 & pyrazine-2-yl & 5 & 82 \\
\hline
\end{tabular}

Table $11 \mathrm{ZnO}$ nanoparticles catalyzed synthesis of benzo[b][1,5]diazepines
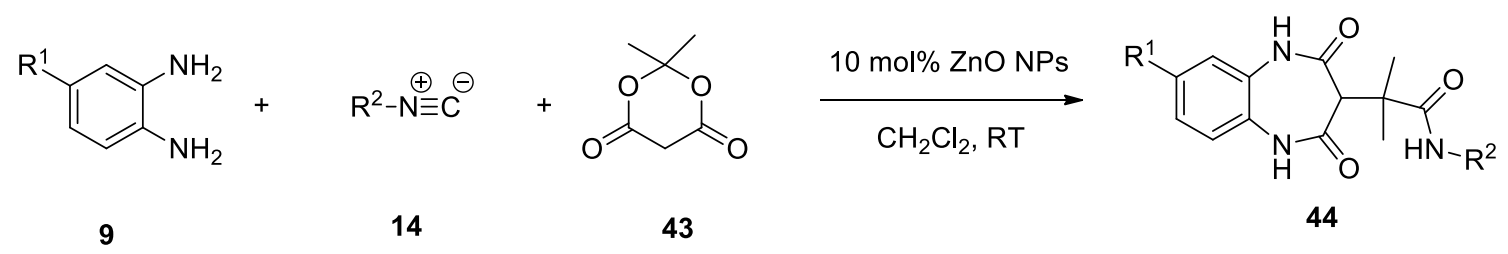

\begin{tabular}{llllllllll}
\hline Entry & $\mathrm{R}^{1}$ & $\mathrm{R}^{2}$ & Time (h) & Yield (\%) & Entry & $\mathrm{R}^{1}$ & $\mathrm{R}^{2}$ & Time (h) & Yield (\%) \\
\hline 1 & $\mathrm{H}$ & cyclohexyl & 3.5 & 93 & 6 & $\mathrm{CH}_{3}$ & cyclohexyl & 3 & 95 \\
2 & $\mathrm{H}$ & $\mathrm{C}\left(\mathrm{CH}_{3}\right)_{3}$ & 3 & 95 & 7 & $\mathrm{CH}_{3}$ & $\mathrm{C}_{\left(\mathrm{CH}_{3}\right)_{3}}$ & 3 & 96 \\
3 & $\mathrm{H}$ & $\mathrm{CH}_{2} \mathrm{C}_{6} \mathrm{H}_{5}$ & 3.5 & 91 & 8 & $\mathrm{CH}_{3}$ & $\mathrm{CH}_{2} \mathrm{C}_{6} \mathrm{H}_{5}$ & 3.5 & 94 \\
4 & $\mathrm{H}$ & $\mathrm{CH}_{2}\left(\mathrm{CH}_{2}\right)_{3} \mathrm{CH}_{3}$ & 3.5 & 92 & 9 & $\mathrm{CH}_{3}$ & $\mathrm{CH}_{2}\left(\mathrm{CH}_{2}\right)_{3} \mathrm{CH}_{3}$ & 3.5 & 95 \\
5 & $\mathrm{H}$ & $4-\mathrm{OCH}_{3} \mathrm{C}_{6} \mathrm{H}_{4}$ & 4 & 91 & 10 & $\mathrm{CH}_{3}$ & $4-\mathrm{OCH}_{3} \mathrm{C}_{6} \mathrm{H}_{4}$ & 3.5 & 93 \\
\hline
\end{tabular}


aromatic aldehydes (5), dimethylacetylenedicarboxylate (2a) and substituted anilines (20) using nano- $\mathrm{ZnO}$ as an efficient, reusable, heterogeneous catalyst in aqueous ethanol at $90{ }^{\circ} \mathrm{C}$ (Table 12). After completion of the reaction, $\mathrm{ZnO}$ nanoparticles were recovered and reused several times without apparent loss in its catalytic efficacy. Safaei-Ghomi et al. [104] demonstrated a $\mathrm{ZnO}$-nanoparticles catalyzed simple and efficient method for the convenient synthesis of 2,3-disubstituted benzo[ $b]$ furans (49) via a one-pot three-component coupling reaction between substituted salisaldehydes (46), secondary amines (47) and phenylacetylene (48) in aqueous ethanol under reflux conditions. $\mathrm{ZnO}$ nanoparicles was found to be more efficient than the other bulk metal oxides such as $\mathrm{MgO}, \mathrm{CuO}, \mathrm{Fe}_{2} \mathrm{O}_{3}$ etc (Table 13).

\section{Synthesis of pyran derivatives}

Pyrans and pyran-annulated heterocyclic scaffolds possess a broad spectrum of significant biological activities that include anticancer, cytotoxic, anti-HIV, anti-inflammatory, antimalarial, antimicrobial activity [105-107]. Das and his group reported a simple, efficient, environmentally benign protocol for the synthesis of 2-amino-3-cyano-4H-pyran derivatives via a one-pot three-component coupling reaction between a series of aromatic aldehydes (5), malononitrile (18) and 1,3-dicarbonyl compounds $(\mathbf{1 7 , 5 0})$ using nano- $\mathrm{ZnO}$ as the catalyst in water at room temperature [108] (Scheme 6). Under the same optimized condition they also synthesized 2-amino-3-cyano- $4 H$-chromenes (52) in good

Table $12 \mathrm{ZnO}$ nanoparticles catalyzed synthesis of 3,4,5-trisubstituted furan-2(5H)-ones<smiles>O=CC1=CC=[Ru]C=C1</smiles>

5<smiles>[CH2+]C#CC(=O)OC</smiles>

2a<smiles></smiles>

20

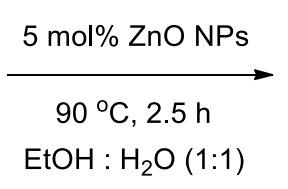

EtOH : $\mathrm{H}_{2} \mathrm{O}(1: 1)$<smiles>[R]c1cccc(NC2=C(C(=O)OC)C(c3ccccc3)OC2=O)c1</smiles>

45

\begin{tabular}{llllllll}
\hline Entry & $\mathrm{R}$ & $\mathrm{R}^{1}$ & Yield (\%) & Entry & $\mathrm{Q}$ & $\mathrm{R}^{1}$ & Yield (\%) \\
\hline 1 & $\mathrm{H}$ & $\mathrm{H}$ & 94 & 7 & $3-\mathrm{OCH}_{3}$ & $\mathrm{H}$ & 85 \\
2 & $\mathrm{H}$ & $4-\mathrm{CH}_{3}$ & 95 & 8 & $4-\mathrm{CH}_{3}$ & $\mathrm{H}$ & 84 \\
3 & $4-\mathrm{OCH}_{3}$ & $\mathrm{H}$ & 88 & 9 & $\mathrm{H}$ & $4-\mathrm{CH}\left(\mathrm{CH}_{3}\right)_{2}$ & 88 \\
4 & $\mathrm{H}$ & $4-\mathrm{F}$ & 84 & 10 & $\mathrm{H}$ & $2-\mathrm{F}$ & 84 \\
5 & $4-\mathrm{Cl}$ & $\mathrm{H}$ & 89 & 11 & $2,4-\mathrm{diCl}$ & $2-\mathrm{F}$ & 85 \\
6 & $2-\mathrm{Cl}$ & $\mathrm{H}$ & 87 & 12 & $2,4-\left(\mathrm{OCH}_{3}\right)_{2}$ & $\mathrm{H}$ & 83 \\
\hline
\end{tabular}

Table $13 \mathrm{ZnO}$ nanoparticles catalyzed synthesis of benzo[b]furans<smiles>[X]c1ccc(O)c(C=O)c1</smiles>

46<smiles>[R]CCCCCN[R1]</smiles>

47<smiles>C#Cc1ccccc1</smiles>

48

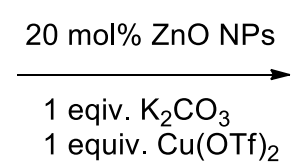

$\mathrm{H}_{2} \mathrm{O}: \mathrm{EtOH}(1: 1)$ reflux<smiles></smiles>

49

\begin{tabular}{llllllllll}
\hline Entry & $\mathrm{X}$ & $\mathrm{R}^{1}$ and $\mathrm{R}^{2}$ & Time $(\mathrm{min})$ & Yield $(\%)$ & Entry & $\mathrm{X}$ & $\mathrm{R}^{1}$ and $\mathrm{R}^{2}$ & Time $(\mathrm{min})$ & Yield $(\%)$ \\
\hline 1 & $\mathrm{H}$ & Morpholine & 90 & 92 & 7 & $\mathrm{Cl}$ & Morpholine & 60 & 92 \\
2 & $\mathrm{H}$ & Piperidine & 90 & 90 & 8 & $\mathrm{Cl}$ & Piperidine & 65 & 94 \\
3 & $\mathrm{H}$ & Dibenzyl & 110 & 80 & 9 & $\mathrm{Cl}$ & Dibenzyl & 80 & 85 \\
4 & $\mathrm{Br}$ & Morpholine & 70 & 94 & 10 & $\mathrm{NO}_{2}$ & Morpholine & 55 & 96 \\
5 & $\mathrm{Br}$ & Piperidine & 75 & 94 & 11 & $\mathrm{NO}_{2}$ & Piperidine & 55 & 94 \\
6 & $\mathrm{Br}$ & Dibenzyl & 90 & 85 & 12 & $\mathrm{NO}_{2}$ & Dibenzyl & 65 & 88 \\
\hline
\end{tabular}


yields from the three-component reaction between aldehydes (5), malononitrile (18) and dimedone (16) (Scheme 6).

Very recently, in 2017, Zavar [109] has also synthesized a series of 2-amino-3-cyano- $4 H$-chromenes (52) within just 10 min using nano- $\mathrm{ZnO}$ as catalyst in ethanol under reflux conditions (Table 14).

\section{Synthesis of xanthenes}

Xanthenes, in particular, 1,8-dioxo-octahydroxanthene moieties, have received significant attention due to their potent pharmacological efficacies such as antimicrobial, anticancer and enzyme inhibitory activity [110-112]. Recently, Safaei-Ghomi et al. have synthesized a variety of structurally diverse xanthene derivatives using $\mathrm{ZnO}$-nanoparticles as catalyst. In 2013, they developed a simple protocol for the efficient synthesis of 1,8-dioxooctahydroxanthene derivatives (53) via a one-pot pseudo three-component condensation between various aldehydes (5) and dimedone (16) in the presence of catalytic amount of $\mathrm{ZnO}$-nanoparticles at $90{ }^{\circ} \mathrm{C}$ under solvent-free conditions [113] (Scheme 7). The optimized reaction conditions also worked satisfactorily in synthesizing a variety of $N$-aryl-1,8-dioxodecahydroacridine derivatives (54) in one-pot when the reaction was carried out in presence of aromatic amines (20) with excellent yield of 70-91\% within just 5-25 min (Scheme 7). After completion of reaction, nano- $\mathrm{ZnO}$ was successfully recovered and recycled for five successive runs with little loss in the catalytic activity.

To explore the catalytic efficiency of this fascinating catalyst, the same group has also employed nano- $\mathrm{ZnO}$ as catalyst for the synthesis of tetrahydrobenzo[a]xanthen-11-ones (56) via one-pot three-component reactions of aldehydes (5), 2-naphthol (55) and dimedone (16) under solvent-free condition at $120^{\circ} \mathrm{C}$ [114] (Table 15). By changing 4-hydroxycoumarin (57) instead of dimedone (16) in the above-mentioned reaction they were also able to synthesize a range of 7-alkyl$6 H, 7 H$-naphtho[ $\left[1^{\prime}, 2^{\prime}: 5,6\right]$ pyrano[3,2-c]chromen-6-one<smiles>CC1(C)CC(=O)CC(=O)C1</smiles>

16<smiles>[R]C1C(C#N)=C(N)OC2=C1C(=O)CC(C)(C)C2</smiles>

52
$10 \mathrm{~mol} \% \mathrm{ZnO} N P s$

$\mathrm{RT}, \mathrm{H}_{2} \mathrm{O}, 3-4 \mathrm{~h}$

9 entries, $79-91 \%$

$\mathrm{R}=\mathrm{C}_{6} \mathrm{H}_{5}, 4-\mathrm{NO}_{2} \mathrm{C}_{6} \mathrm{H}_{4}, 3-\mathrm{NO}_{2} \mathrm{C}_{6} \mathrm{H}_{4}, 4-\mathrm{F}-\mathrm{C}_{6} \mathrm{H}_{4}, 4-\mathrm{OCH}_{3} \mathrm{C}_{6} \mathrm{H}_{4}$, 4- $\mathrm{CH}_{3} \mathrm{C}_{6} \mathrm{H}_{4}, 4-\mathrm{N}\left(\mathrm{CH}_{3}\right)_{2} \mathrm{C}_{6} \mathrm{H}_{4}$, 2-furyl, 4-pyridyl

Scheme $6 \mathrm{ZnO}$ nanoparticles catalyzed synthesis of $4 H$-pyran derivatives<smiles>[R]C(=O)CC(C)=O</smiles>

$$
\text { 17; } \mathrm{R}^{1}=\mathrm{OC}_{2} \mathrm{H}_{5}
$$$$
\text { 50; } \mathrm{R}^{1}=\mathrm{CH}_{3}
$$

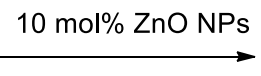

$\mathrm{RT}, \mathrm{H}_{2} \mathrm{O}, 2-4 \mathrm{~h}$

18 entries, $83-98 \%$<smiles>[R]C(=O)C1=C(C)OC(N)=C(C#N)C1[R]</smiles>

51

18

$$
\begin{aligned}
\mathrm{R}= & \mathrm{C}_{6} \mathrm{H}_{5}, 4-\mathrm{NO}_{2} \mathrm{C}_{6} \mathrm{H}_{4}, 3-\mathrm{NO}_{2} \mathrm{C}_{6} \mathrm{H}_{4}, 4-\mathrm{F}-\mathrm{C}_{6} \mathrm{H}_{4}, \\
& 4-\mathrm{Cl}_{-} \mathrm{C}_{6} \mathrm{H}_{4}, \mathrm{CH}_{3} \mathrm{CH}_{2} \mathrm{CH}_{2}, 4-\mathrm{OH}-\mathrm{C}_{6} \mathrm{H}_{4}, \\
& \text { 4-OCH } \mathrm{OCH}_{3} \mathrm{C}_{6} \mathrm{H}_{4}, 4-\mathrm{CH}_{3} \mathrm{C}_{6} \mathrm{H}_{4}, 4-\mathrm{N}\left(\mathrm{CH}_{3}\right)_{2} \mathrm{C}_{6} \mathrm{H}_{4}, \\
& \text { 2-furyl, 4-pyridyl }
\end{aligned}
$$

Table 14 Nano- $\mathrm{ZnO}$ catalyzed synthesis of 2-amino-3-cyano- $4 H$-chromenes

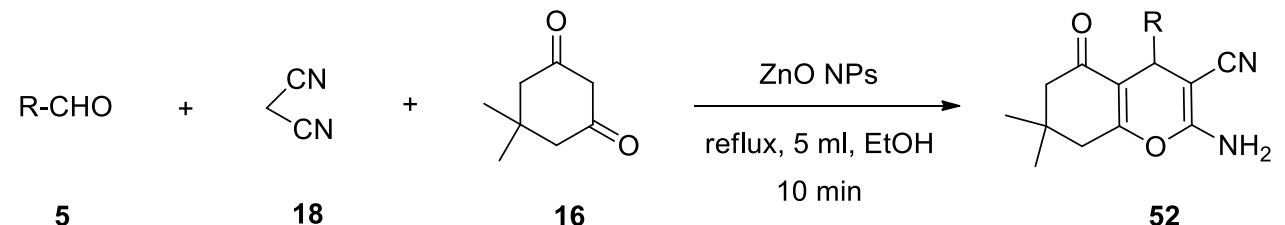

\begin{tabular}{llllll}
\hline Entry & $\mathrm{R}$ & Yield (\%) & Entry & $\mathrm{R}$ & Yield (\%) \\
\hline 1 & $\mathrm{C}_{6} \mathrm{H}_{5}$ & 90 & 6 & $2-\mathrm{NO}_{2} \mathrm{C}_{6} \mathrm{H}_{4}$ & 85 \\
2 & $4-\mathrm{N}\left(\mathrm{CH}_{3}\right)_{2} \mathrm{C}_{6} \mathrm{H}_{4}$ & 80 & 7 & $3-\mathrm{NO}_{2} \mathrm{C}_{6} \mathrm{H}_{4}$ & 92 \\
3 & $4-\mathrm{OCH}_{3} \mathrm{C}_{6} \mathrm{H}_{4}$ & 78 & 8 & $2,4-\mathrm{diClC}_{6} \mathrm{H}_{4}$ & 80 \\
4 & $2-\mathrm{OCH}_{3} \mathrm{C}_{6} \mathrm{H}_{4}$ & 75 & 9 & $4-\mathrm{ClC}_{6} \mathrm{H}_{4}$ & 80 \\
5 & $3,4-\left(\mathrm{OCH}_{3}\right)_{2} \mathrm{C}_{6} \mathrm{H}_{3}$ & 80 & 10 & $4-\mathrm{BrC}_{6} \mathrm{H}_{4}$ & 95 \\
\hline
\end{tabular}




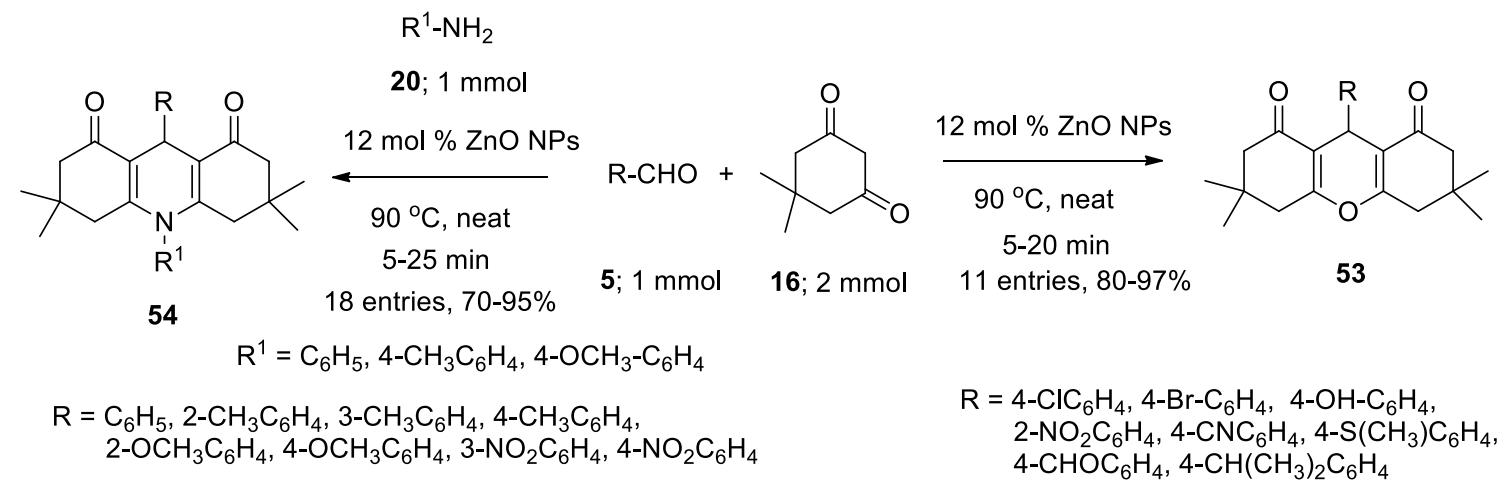

Scheme $7 \mathrm{ZnO}$ nanoparticles catalyzed synthesis of 1,8-dioxo-decahydroacridines and 1,8-dioxooctahydro-xanthenes

Table $15 \mathrm{ZnO}$ nanoparticles catalyzed synthesis of tetrahydrobenzo[ $a]$ xanthen-11-ones

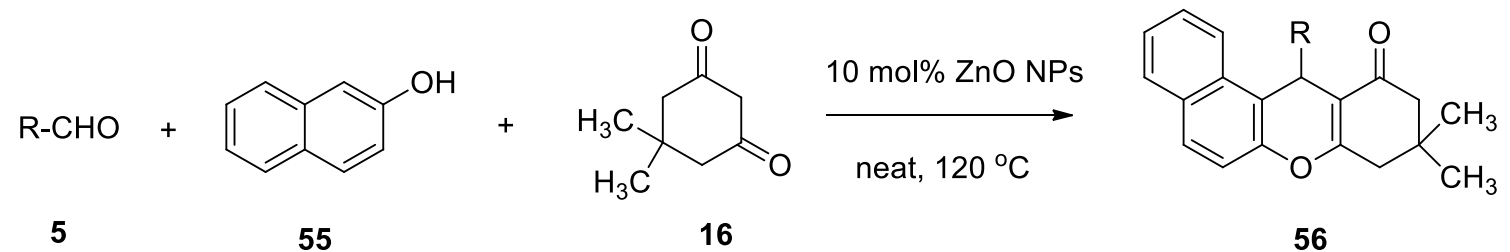

\begin{tabular}{|c|c|c|c|c|c|c|c|}
\hline Entry & $\mathrm{R}$ & Time (min) & Yield (\%) & Entry & $\mathrm{R}$ & Time (min) & Yield (\%) \\
\hline 1 & $\mathrm{C}_{6} \mathrm{H}_{5}$ & 16 & 88 & 9 & $4-\mathrm{CHOC}_{6} \mathrm{H}_{4}$ & 18 & 91 \\
\hline 2 & $3-\mathrm{NO}_{2} \mathrm{C}_{6} \mathrm{H}_{4}$ & 15 & 90 & 10 & $2-\mathrm{S}\left(\mathrm{CH}_{3}\right) \mathrm{C}_{6} \mathrm{H}_{4}$ & 22 & 87 \\
\hline 3 & $4-\mathrm{NO}_{2} \mathrm{C}_{6} \mathrm{H}_{4}$ & 10 & 95 & 11 & $4-\mathrm{CH}\left(\mathrm{CH}_{3}\right)_{2} \mathrm{C}_{6} \mathrm{H}_{4}$ & 20 & 86 \\
\hline 4 & $4-\mathrm{ClC}_{6} \mathrm{H}_{4}$ & 12 & 93 & 12 & $4-\mathrm{OCH}_{3} \mathrm{C}_{6} \mathrm{H}_{4}$ & 22 & 88 \\
\hline 5 & 2,4-diClC ${ }_{6} \mathrm{H}_{3}$ & 20 & 92 & 13 & $2-\mathrm{OCH}_{3} \mathrm{C}_{6} \mathrm{H}_{4}$ & 30 & 85 \\
\hline 6 & $4-\mathrm{BrC}_{6} \mathrm{H}_{4}$ & 15 & 90 & 14 & $4-\mathrm{OHC}_{6} \mathrm{H}_{4}$ & 25 & 90 \\
\hline 7 & $4-\mathrm{FC}_{6} \mathrm{H}_{4}$ & 15 & 92 & 15 & $3-\mathrm{CH}_{3} \mathrm{C}_{6} \mathrm{H}_{4}$ & 22 & 85 \\
\hline 8 & $4-\mathrm{CNC}_{6} \mathrm{H}_{4}$ & 15 & 90 & 16 & 4- $\mathrm{CH}_{3} \mathrm{C}_{6} \mathrm{H}_{4}$ & 18 & 90 \\
\hline
\end{tabular}

Table $16 \mathrm{ZnO}$ nanoparticles catalyzed synthesis of pyrano[3,2-c]chromen derivatives

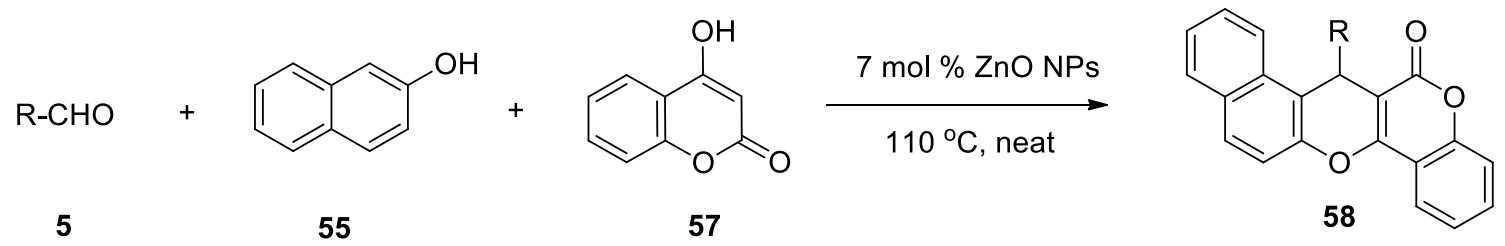

\begin{tabular}{llllllll}
\hline Entry & $\mathrm{R}$ & Time $(\min )$ & Yield $(\%)$ & Entry & $\mathrm{R}$ & Time (min) & Yield $(\%)$ \\
\hline 1 & $\mathrm{C}_{6} \mathrm{H}_{5}$ & 50 & 70 & 7 & $2,4-\mathrm{diClC}_{6} \mathrm{H}_{3}$ & 45 & 85 \\
2 & $4-\mathrm{ClC}_{6} \mathrm{H}_{4}$ & 40 & 91 & 8 & $4-\mathrm{BrC}_{6} \mathrm{H}_{4}$ & 40 & 89 \\
3 & $4-\mathrm{FC}_{6} \mathrm{H}_{4}$ & 40 & 92 & 9 & $4-\mathrm{OCH}_{3} \mathrm{C}_{6} \mathrm{H}_{4}$ & 50 & 80 \\
4 & $4-\mathrm{CH}_{3} \mathrm{C}_{6} \mathrm{H}_{4}$ & 60 & 85 & 10 & $4-\mathrm{OHC}_{6} \mathrm{H}_{4}$ & 60 & 80 \\
5 & $4-\mathrm{NO}_{2} \mathrm{C}_{6} \mathrm{H}_{4}$ & 40 & 93 & 11 & $3,4-\mathrm{diClC}_{6} \mathrm{H}_{3}$ & 60 \\
6 & $3-\mathrm{NO}_{2} \mathrm{C}_{6} \mathrm{H}_{4}$ & 60 & 70 & 12 & $2,5-\left(\mathrm{OCH}_{3}\right)_{2} \mathrm{C}_{6} \mathrm{H}_{3}$ & 60 & 81 \\
\hline
\end{tabular}

derivatives (58) using the same nano- $\mathrm{ZnO}$ as catalyst under solvent-free condition at $110{ }^{\circ} \mathrm{C}$ [115] (Table 16). Short reaction times, high yields, easy workup procedure, wide substrate tolerance, small catalyst loading, reusability of the catalyst and solvent-free conditions are some of the salient features of these developed protocols. 
Table $17 \mathrm{ZnO}$ nanoparticles catalyzed synthesis of coumarins

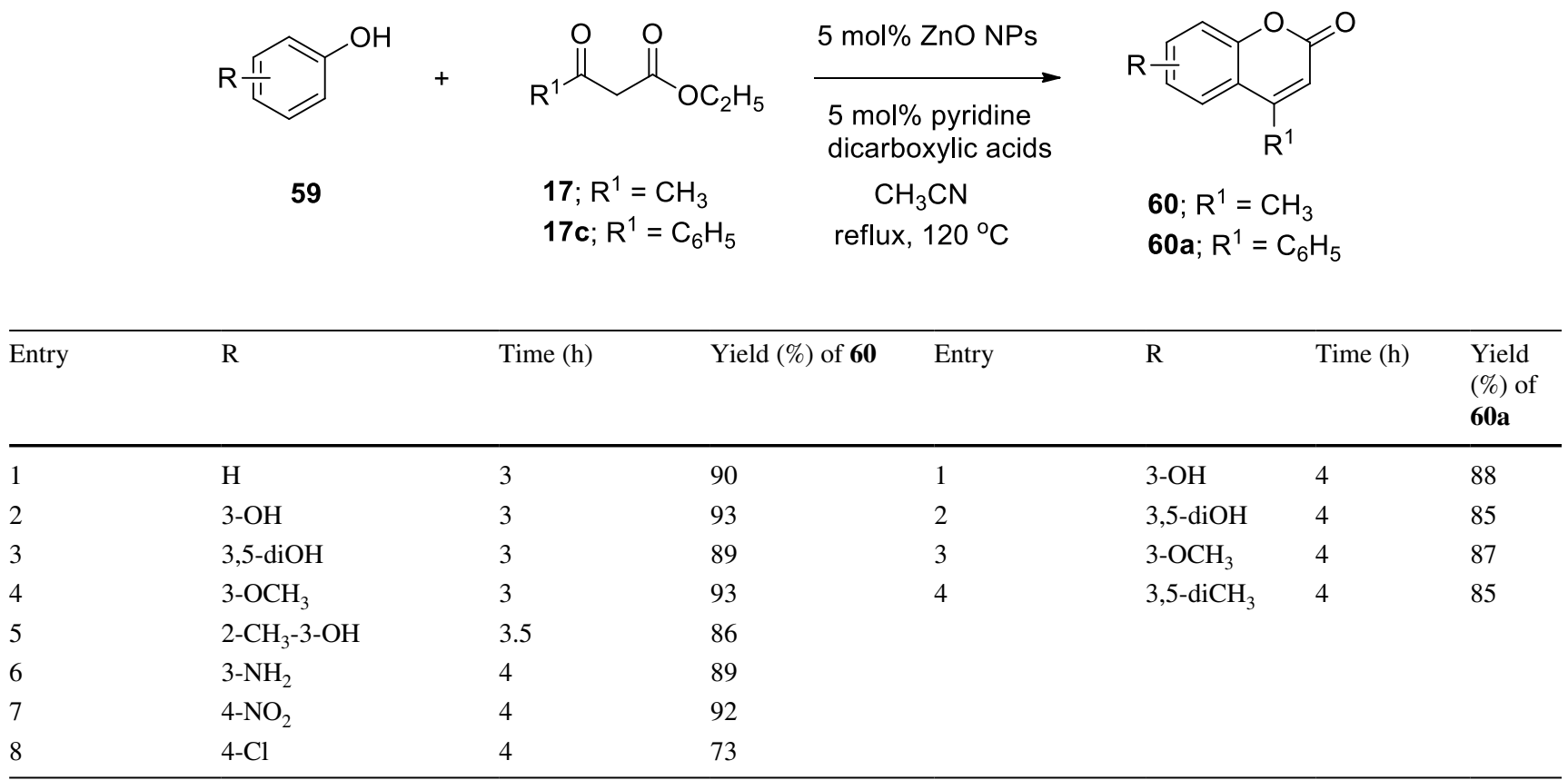

\section{Synthesis of coumarins}

Coumarins are very common in the naturally occurring heterocycles like warfarin, phenprocoumon, coumatetralyl, carbochromen, bromadialone, etc. Heterocycles containing this important structural motif exhibit a wide range of pharmaceutical activities that include antibacterial, antiHIV, antiviral, anticoagulant, antioxidant and anticancer activities [116-120]. Goswami [121] synthesized a wide range of 4 -substituted coumarins ( 60 ) by the reaction of a wide range of structurally diverse phenols (59) and ethyl acetoacetate (17) or ethyl benzoyl acetate (17c) in the presence of nanocrystalline $\mathrm{ZnO}$ as catalyst and pyridine dicarboxylic acid as co-catalyst in acetonitrile under reflux conditions (Table 17).

Kumar et al. [122] achieved the synthesis of various 3 -substituted coumarins (60b) by the nano- $\mathrm{ZnO}$ catalyzed reactions between salisaldehydes (46) and various 1,3-dicarbonyl compounds $(\mathbf{1 7 , 1 8 a}, \mathbf{6 1})$ under microwave irradiation at $120{ }^{\circ} \mathrm{C}$ (Table 18). Under the same optimized reaction conditions they also synthesized benzo[f] chromen-3-ones (63) by using 2-hydroxy naphthaldehyde (62) instead of salisaldehyde (46) (Table 18). After completion of reaction, nano- $\mathrm{ZnO}$ was successfully recovered and recycled for several runs with consistent catalytic activity. During optimization, $\mathrm{ZnO}$ nanoparicles were found to be much more efficient than the commercially available bulk $\mathrm{ZnO}$. Das and his group employed nanocrystalline $\mathrm{ZnO}$ as an efficient, reusable catalyst for the one-pot green synthesis of a series of benzylamino coumarin derivatives (64) from the reactions of various aromatic aldehydes (5), 4-hydroxycoumarin (57) and cyclic secondary amines (47) in water at room temperature [123] (Scheme 8).

\section{Synthesis of highly functionalized $4 \mathrm{H}$-chromenes}

Das and his group developed another nano- $\mathrm{ZnO}$ catalyzed protocol for the efficient synthesis of dihydropyrano[2,3$c$ ]chromenes (66) via a one-pot, three-component coupling reaction between aromatic aldehydes (5), malononitrile (18) and 3-hydroxycoumarin $(\mathbf{6 5})$ in water at $70{ }^{\circ} \mathrm{C}$ [124] (Table 19). Later on, the same group also synthesized a series of structurally diverse $4 H$-chromene derivatives (69) by the reactions of salisaldehydes (46), active methylene compounds $(\mathbf{1 6}, \mathbf{1 6 a}, \mathbf{1 8}, \mathbf{6 7} \mathbf{a})$ and $\mathrm{C}-\mathrm{H}$ activated nucleophiles $(\mathbf{2 8}, 55,57,68 \mathrm{a}, 68 \mathrm{~b}, \mathbf{6 8 c}, \mathbf{6 8 d})$ employing the same nano- $\mathrm{ZnO}$ as catalyst in aqueous medium at $55^{\circ} \mathrm{C}$ [125] (Scheme 9). During optimization it was found that the catalytic activity of nano- $\mathrm{ZnO}$ is superior to the other nano metal oxides such as nano- $\mathrm{Al}_{2} \mathrm{O}_{3}$, nano- $\mathrm{MgO}$ tested for these reactions. Nano-ZnO was recovered easily and recycled six times without significant loss in catalytic activity. 
Table $18 \mathrm{ZnO}$ nanoparticles catalyzed synthesis of coumarins<smiles>O=Cc1c(O)ccc2ccccc12</smiles>

62<smiles>[R]c1cc2c(ccc3ccccc32)oc1=O</smiles>

63
$10 \mathrm{~mol} \% \mathrm{ZnO}$ NPs

$\mathrm{MW}, 120^{\circ} \mathrm{C}$

2 entries

$\mathrm{R}^{1}=\mathrm{COCH}_{3} ; 6 \min , 92 \%$

$\mathrm{R}^{1}=\mathrm{CN} ; 5 \mathrm{~min}, 91 \%$<smiles>[R]CC(=O)OCC</smiles>

$\mathrm{MW}, 120^{\circ} \mathrm{C}$

61; $\mathrm{R}^{1}=\mathrm{COOC}_{2} \mathrm{H}_{5}$

17; $\mathrm{R}^{1}=\mathrm{COCH}_{3}$

18a; $R^{1}=C N$<smiles>O=Cc1cc[R]#cc1O</smiles>

46<smiles>[R]c1cc2cc[R17]cc2oc1=O</smiles>

$60 \mathrm{~b}$

\begin{tabular}{lllll}
\hline Entry & $\mathrm{R}$ & $\mathrm{R}^{1}$ & Time (min) & $\begin{array}{l}\text { Yield } \\
\text { (\%) of } \\
\text { 60b }\end{array}$ \\
\hline 1 & & & 92 \\
2 & $\mathrm{H}$ & $\mathrm{COOC}_{2} \mathrm{H}_{5}$ & 5 & 90 \\
3 & $\mathrm{H}$ & $\mathrm{COCH}_{3}$ & 6 & 86 \\
4 & $\mathrm{COOC}_{2} \mathrm{H}_{5}$ & 4 & 92 \\
5 & $3,4-(\mathrm{OH})_{2}$ & 5 & 90 \\
6 & $3-\mathrm{OCH}_{3}$ & $\mathrm{COOC}_{2} \mathrm{H}_{5}$ & 5 & 92 \\
7 & $3-\mathrm{OCH}_{3}$ & $\mathrm{COCH}_{3}$ & 6 & 87 \\
8 & $4-\mathrm{OCH}_{3}$ & $\mathrm{COOC}_{2} \mathrm{H}_{5}$ & 5 & 88 \\
9 & $4-\mathrm{OCH}_{3}$ & $\mathrm{COCH}_{3}$ & 5 & 90 \\
10 & $3-\mathrm{N}\left(\mathrm{C}_{2} \mathrm{H}_{5}\right)_{2}$ & $\mathrm{COOC}_{2} \mathrm{H}_{5}$ & 7 & 62 \\
11 & $3-\mathrm{N}\left(\mathrm{C}_{2} \mathrm{H}_{5}\right)_{2}$ & $\mathrm{COCH}_{3}$ & 7 & 85 \\
12 & $3-\mathrm{N}\left(\mathrm{C}_{2} \mathrm{H}_{5}\right)_{2}$ & $\mathrm{CN}_{3}$ & 5 & 92 \\
13 & $3-\mathrm{OH}$ & $\mathrm{COOC}_{2} \mathrm{H}_{5}$ & 7 & 95 \\
14 & $4-\mathrm{OH}$ & $\mathrm{COOC}_{2} \mathrm{H}_{5}$ & 9 & 93 \\
15 & $5-\mathrm{Cl}$ & 6 & 93 \\
16 & $5-\mathrm{Br}$ & $\mathrm{COCH}_{3}$ & 8 & 6 \\
\hline
\end{tabular}

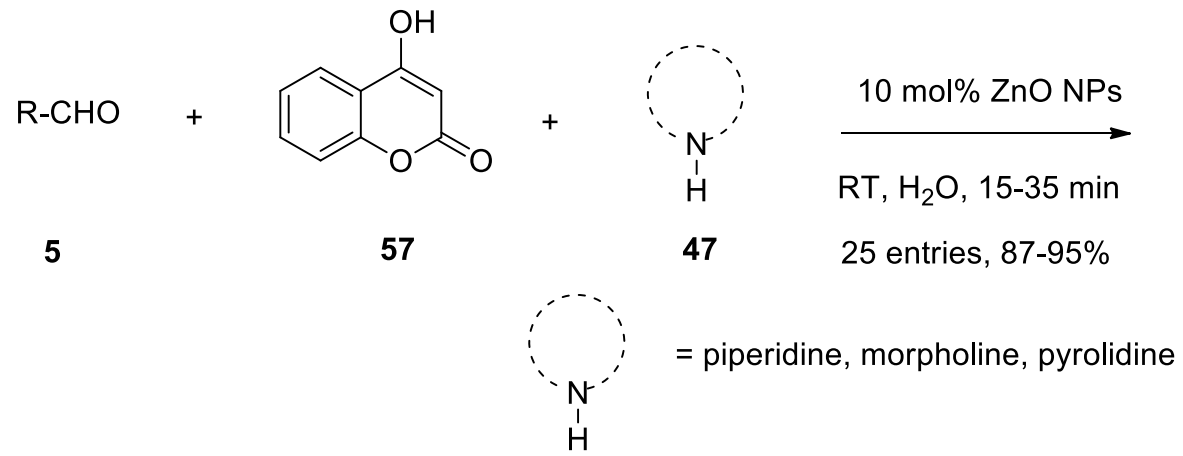<smiles>[R]C(c1c(O)c2ccccc2oc1=O)N1CCCCCC1</smiles>

64

$\mathrm{R}=\mathrm{C}_{6} \mathrm{H}_{5}, 3-\mathrm{NO}_{2} \mathrm{C}_{6} \mathrm{H}_{4}, 4-\mathrm{ClC}_{6} \mathrm{H}_{4}, 4-\mathrm{FC}_{6} \mathrm{H}_{4}, 3-\mathrm{OCH}_{3} \mathrm{C}_{6} \mathrm{H}_{4}, 4-$ pyridyl, 4- $\mathrm{NO}_{2} \mathrm{C}_{6} \mathrm{H}_{4}$, 4- $\mathrm{CH}_{3} \mathrm{C}_{6} \mathrm{H}_{4}, 4-\mathrm{OCH}_{3}-3-\mathrm{OHC}_{6} \mathrm{H}_{3}, 2,3-\left(\mathrm{CH}_{3}\right)_{2} \mathrm{C}_{6} \mathrm{H}_{3}$

Scheme $8 \mathrm{ZnO}$ nanoparticles catalyzed synthesis of benzylamino coumarin derivatives 
Table $19 \mathrm{ZnO}$ nanoparticles catalyzed synthesis of dihydropyrano[2,3-c]chromenes

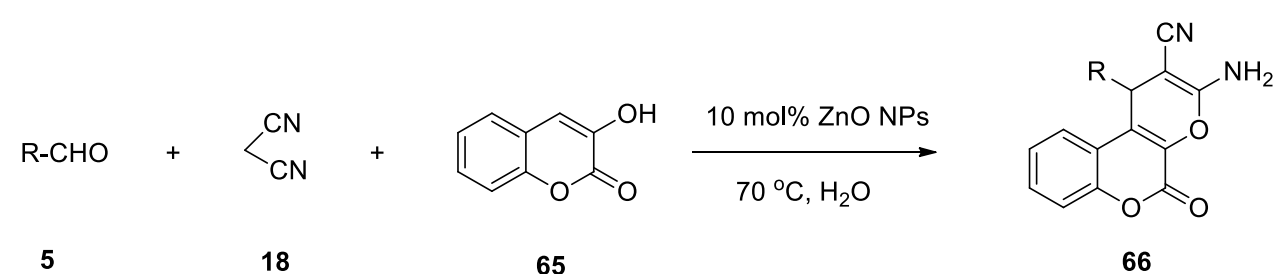

\begin{tabular}{llllllll}
\hline Entry & $\mathrm{R}$ & Time (h) & Yield (\%) & Entry & $\mathrm{R}$ & Time (h) \\
\hline 1 & $\mathrm{C}_{6} \mathrm{H}_{5}$ & 3 & 87 & 6 & Yield (\%) \\
2 & $4-\mathrm{NO}_{2} \mathrm{C}_{6} \mathrm{H}_{4}$ & 2.5 & 91 & 7 & 4-naphthyl & 2.5 & 89 \\
3 & $4-\mathrm{OCH}_{6} \mathrm{C}_{4} \mathrm{H}_{4}$ & 3 & 78 & 8 & 4-CNC $\mathrm{H}_{4}$ & 2.5 & 90 \\
4 & $4-\mathrm{BrC}_{6} \mathrm{H}_{4}$ & 2.5 & 89 & 9 & 2-thienyl & 3 & 90 \\
5 & $4-\mathrm{CH}_{3} \mathrm{C}_{6} \mathrm{H}_{3}$ & 3 & 83 & 10 & 4-CHOC ${ }_{6} \mathrm{H}_{4}$ & 4 & 75 \\
\hline
\end{tabular}

\section{Nano-ZnO catalyzed synthesis of $\mathrm{N}$ - as well as $\boldsymbol{O}$-heterocycles}

\section{Synthesis of 6-amino-5-cyano-pyrano[2,3-c] pyrazoles}

Pyrano[2,3-c]pyrazole and its derivatives possess significant biological efficacies that include anti-inflammatory, molluscicidal, insecticidal, antitumor and anticancer activity [126, 127]. Tekale et al. [128] developed a simple, convenient and practical method for the efficient synthesis of 6-amino5-cyano-pyrano[2,3-c]pyrazoles (71) via a four-component reaction of ethyl acetoacetate (17), hydrazine hydrate (70), malononitrile (18) and various aromatic aldehydes (5) using nano- $\mathrm{ZnO}$ as a recyclable heterogeneous catalyst in aqueous medium at $70{ }^{\circ} \mathrm{C}$ (Table 20). In the same year, from the same batch of reactions, Sachdeva et al. [129] replaced malononirile (18) by ethylcyano acetate (18a) and synthesized ethyl 6-amino-pyrano[2,3-c]pyrazoles-5-carboxylate derivatives (71a) in good yields using the same nano- $\mathrm{ZnO}$ as a reusable catalyst in aqueous medium at room temperature (Table 21).

\section{Synthesis of pyrazole based pyrido[2,3-d] pyrimidine-diones}

Heravi et al. [130] reported the efficient synthesis of a series of pyrazolo- $\left[4^{\prime}, 3^{\prime}: 5,6\right]$ pyrido[2,3- $\left.d\right]$ pyrimidine-dione derivatives (72) via a one-pot five-component condensation between hydrazine hydrate (70), ethyl acetoacetate (17), 1,3-dimethyl barbituric acid (67a), aromatic aldehydes (5) and ammonium acetate (7) in the presence of nano-ZnO in water under reflux conditions (Table 22). Use of nano-ZnO as catalyst offers several advantages such as operational simplicity, wide range of substrate tolerance, easy work-up and high yields of products.

\section{Synthesis of structurally diverse pyridine derivatives}

Siddiqui et al. [131] demonstrated the catalytic efficiency of $\mathrm{ZnO}$-nanoparticles for the efficient synthesis of a series of novel pyridine derivatives $(\mathbf{7 6}, \mathbf{7 7}, \mathbf{7 8 , 7 9 , 8 0})$ via a sequential three-component reaction of $\beta$-enaminones (73), ammonium acetate (7) and various active methylene compounds $(\mathbf{1 6}, 17,17 \mathbf{a}, 43,57,67 \mathbf{a}, 67 \mathbf{a a}, 74)$ under solvent-free conditions at $70{ }^{\circ} \mathrm{C}$. Nano-ZnO was recovered easily and recycled six times without significant loss in catalytic activity (Scheme 10).

\section{Synthesis of 1,3-oxazoline-2-thione derivatives}

Haerizade et al. [132] synthesized a series of functionalized 2-thioxo-2,3-dihydro-1,3-oxazoles (83) from one-pot three-component reactions of ammonium thiocyanate $(\mathbf{8 1})$, various acid chlorides $(\mathbf{8 2})$ and phenacyl bromides $(\mathbf{3})$ in the presence of a catalytic amount of nano- $\mathrm{ZnO}$ and $\mathrm{N}$-methylimidazole as co-catalyst under solvent-free conditions at room temperature (Table 23).

\section{Supported nano-ZnO catalyzed heterocycles synthesis}

\section{Synthesis of thieno[2,3-c]pyridine derivatives}

Sangshetti et al. [133] employed combined nano zinc oxide and titanium dioxide [nano $\left.\left(\mathrm{ZnO}-\mathrm{TiO}_{2}\right)\right]$ as an efficient catalytic system for the synthesis of a series of novel 4,5,6,7-tetrahydro-6-((5-substituted-1,3,4-oxadiazol-2-yl) methyl)thieno[2,3-c]pyridines (85) from the reactions of 2-(4,5-dihydrothieno[2,3-c]pyridin-6(7H)-yl)acetohydrazide 


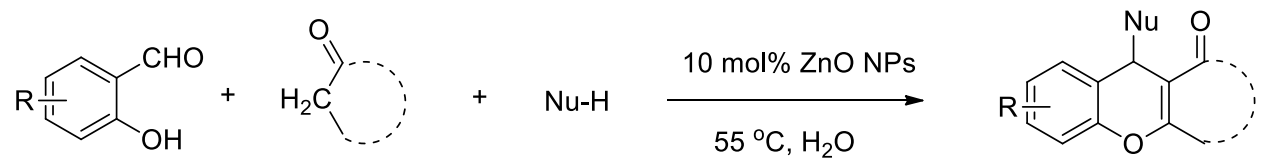

46

67

68

69<smiles>O=C1CCCCC1</smiles>

$\mathrm{Nu}-\mathrm{H}=4$-hydroxycoumarin (57), 4-aminocoumarin (68a), 2-naphthol (55), 5-methyl-3H-pyrazol-3-one (68b) 5-methyl-2-phenyl-1 $\mathrm{H}$-pyrazol-3(2H)-one (68c), indole (68d), 6-aminouracil (28)<smiles>CC1(C)CC(=O)C2=C(C1)Oc1cc[R][c]c1C2c1c(O)c2ccccc2oc1=O</smiles>

69a; $\mathrm{R}=\mathrm{H} ; 45 \mathrm{~min}, 92 \%$

69b; $\mathrm{R}=5-\mathrm{NO}_{2}, 40 \mathrm{~min}, 90 \%$

69c; $\mathrm{R}=4-\mathrm{OCH}_{3}, 45 \mathrm{~min}, 91 \%$<smiles></smiles>

69d; $\mathrm{R}^{1}=\mathrm{C}\left(\mathrm{CH}_{3}\right)_{2} ; 45 \mathrm{~min}, 90 \%$

$69 \mathrm{e} ; \mathrm{R}^{1}=\mathrm{CH}_{2} ; 45 \mathrm{~min}, 90 \%$<smiles>O=C1CCCC2=C1C(c1c(O)c3ccccc3oc1=O)c1ccccc1O2</smiles>

69f; $45 \mathrm{~min}, 91 \%$<smiles>[X]c1c(C2c3ccccc3Oc3c2c(=O)n(C)c(=O)n3C)c2ccccc2oc1=O</smiles>

69g; $\mathrm{X}=\mathrm{OH} ; 40 \mathrm{~min}, 91 \%$ $69 \mathbf{h} ; \mathrm{X}=\mathrm{NH}_{2}, 50 \mathrm{~min}, 91 \%$<smiles>N#CC1=C(N)Oc2ccccc2C1c1c(O)c2ccccc2oc1=O</smiles>

$69 i ; 40 \min , 87 \%$<smiles>Cn1c2c(c(=O)n(C)c1=O)C(c1c[nH]c3ccccc13)c1ccccc1O2</smiles>

69n; $50 \mathrm{~min}, 91 \%$<smiles></smiles><smiles>[R]CCC(=O)C1=C(C[R1])Oc2ccccc2C1c1c(O)ccc2ccccc12</smiles>

69j; $\mathrm{R}^{1}=\mathrm{C}\left(\mathrm{CH}_{3}\right)_{2} ; 55 \mathrm{~min}, 93 \%$ $69 k$; $\mathrm{R}^{1}=\mathrm{CH}_{2} ; 45 \mathrm{~min}, 91 \%$<smiles>N#CC1=C(N)Oc2ccccc2C1c1c[nH]c2ccccc12</smiles>

69o; $40 \mathrm{~min}, 90 \%$<smiles>CC1(C)CC(=O)C2=C(C1)Oc1ccccc1C2c1c(N)[nH]c(=O)[nH]c1=O</smiles>

$69 u$; $60 \mathrm{~min}, 86 \%$<smiles>Cn1c2c(c(=O)n(C)c1=O)C(c1c(O)ccc3ccccc13)c1ccccc1O2</smiles>

69I; $50 \mathrm{~min}, 92 \%$<smiles>CC1(C)CC(=O)C2=C(C1)Oc1ccccc1C2c1c[nH]c2ccccc12</smiles>

69p; $50 \mathrm{~min}, 91 \%$<smiles>Cn1c2c(c(=O)n(C)c1=O)C(c1c(N)[nH]c(=O)[nH]c1=O)c1ccccc1O2</smiles>

$69 v$; $65 \mathrm{~min}, 85 \%$

69q; $\mathrm{R}^{1}=\mathrm{C}\left(\mathrm{CH}_{3}\right)_{2} ; 50 \mathrm{~min}, 88 \%$ 69s; $\mathrm{R}^{1}=\mathrm{C}\left(\mathrm{CH}_{3}\right)_{2} ; 50 \mathrm{~min}, 90 \%$ 69r; $\mathrm{R}^{1}=\mathrm{CH}_{2} ; 50 \mathrm{~min}, 90 \%$

69t; $\mathrm{R}^{1}=\mathrm{CH}_{2} ; 40 \mathrm{~min}, 89 \%$

Scheme $9 \mathrm{ZnO}$ nanoparticles catalyzed synthesis of densely functionalized $4 H$-chromenes

(84) and various substituted aldehydes (5) in ethanol under reflux conditions (Table 24). Catalytic activity of nano $\left(\mathrm{ZnO}-\mathrm{TiO}_{2}\right)$ was found to be superior to the individual effect of nano $\mathrm{ZnO}$ or nano $\mathrm{TiO}_{2}$. All the synthesized compounds were screened for their antimicrobial activities. Among them compounds 85f, 85k, 85I have promising antibacterial as well as antifungal efficacies whereas compound $\mathbf{8 5} \mathbf{j}$ possess promising antibacterial activity. 
Table $20 \mathrm{ZnO}$ nanoparticles catalyzed synthesis of 6-amino-5-cyano-pyrano[2,3-c]pyrazoles

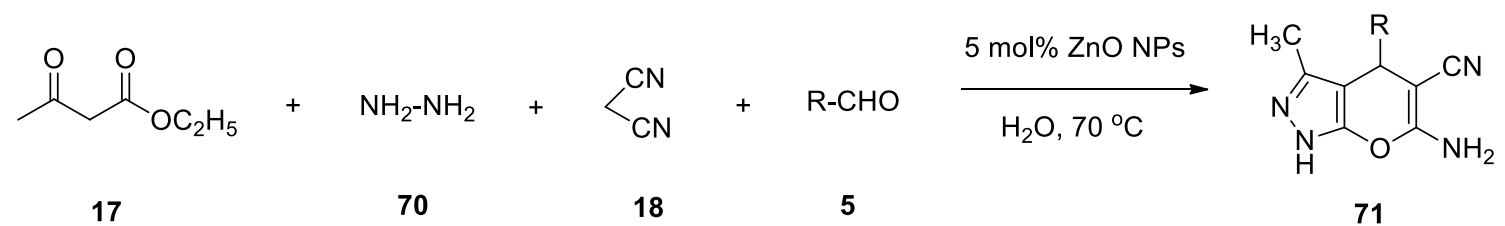

\begin{tabular}{|c|c|c|c|c|c|c|c|}
\hline Entry & $\mathrm{R}$ & Time (min) & Yield (\%) & Entry & $\mathrm{R}$ & Time (min) & Yield (\%) \\
\hline 1 & $\mathrm{C}_{6} \mathrm{H}_{5}$ & 60 & 94 & 8 & 4- $\mathrm{BrC}_{6} \mathrm{H}_{4}$ & 65 & 85 \\
\hline 2 & $4-\mathrm{ClC}_{6} \mathrm{H}_{4}$ & 60 & 90 & 9 & $4-\mathrm{NO}_{2} \mathrm{C}_{6} \mathrm{H}_{4}$ & 90 & 87 \\
\hline 3 & $4-\mathrm{N}\left(\mathrm{CH}_{3}\right)_{2} \mathrm{C}_{6} \mathrm{H}_{4}$ & 70 & 86 & 10 & $4-\mathrm{OCH}_{3} \mathrm{C}_{6} \mathrm{H}_{4}$ & 70 & 90 \\
\hline 4 & 4-S $\left(\mathrm{CH}_{3}\right) \mathrm{C}_{6} \mathrm{H}_{4}$ & 70 & 88 & 11 & $4-\mathrm{NO}_{2} \mathrm{C}_{6} \mathrm{H}_{4}$ & 90 & 87 \\
\hline 5 & $4-\mathrm{OH}-\mathrm{C}_{6} \mathrm{H}_{4}$ & 90 & 82 & 12 & 2-furyl & 80 & 86 \\
\hline 6 & $2-\mathrm{ClC}_{6} \mathrm{H}_{4}$ & 80 & 89 & 13 & $4-\mathrm{OH}-3-\mathrm{OCH}_{3} \mathrm{C}_{6} \mathrm{H}_{3}$ & 80 & 91 \\
\hline 7 & $4-\mathrm{CH}_{3} \mathrm{C}_{6} \mathrm{H}_{4}$ & 70 & 90 & & & & \\
\hline
\end{tabular}

Table $21 \mathrm{ZnO}$ nanoparticles catalyzed synthesis of ethyl 6-amino-pyrano[2,3-c]pyrazoles-5-carboxylate derivatives

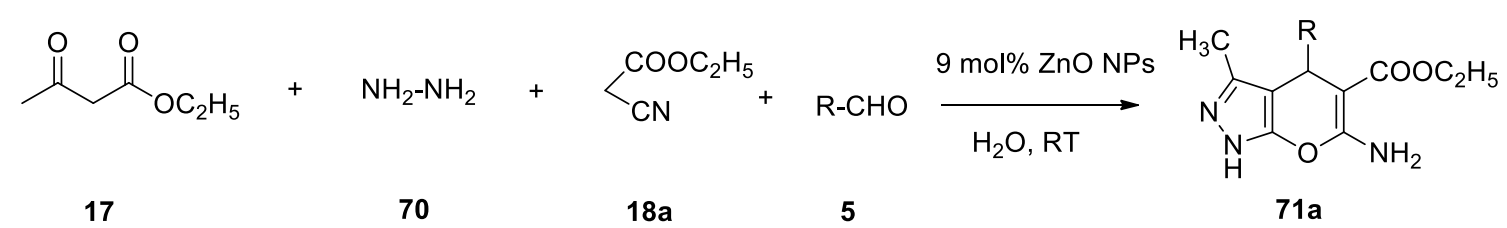

\begin{tabular}{llllllll}
\hline Entry & $\mathrm{R}$ & Time $(\mathrm{min})$ & Yield $(\%)$ & Entry & $\mathrm{R}$ & Time (min) & Yield (\%) \\
\hline 1 & $3,4-\left(\mathrm{OCH}_{3}\right)_{2} \mathrm{C}_{6} \mathrm{H}_{3}$ & 60 & 90 & 6 & 3- $\mathrm{CH}_{3}$-2-furyl & 60 & 86 \\
2 & $3-\mathrm{OCH}_{3} \mathrm{C}_{6} \mathrm{H}_{4}$ & 55 & 85 & 7 & 2-thienyl & 55 & 87 \\
3 & $3,4,5-\left(\mathrm{OCH}_{3}\right)_{3} \mathrm{C}_{6} \mathrm{H}_{2}$ & 55 & 86 & 8 & 3-pyridyl & 60 & 85 \\
4 & $4-\mathrm{ClC}_{6} \mathrm{H}_{4}$ & 60 & 87 & 9 & 2-OH-C $\mathrm{H}_{4}$ & 60 & 87 \\
5 & $4-\mathrm{OCH}_{3} \mathrm{C}_{6} \mathrm{H}_{4}$ & 55 & 89 & 10 & 3-OH-4-OCH $\mathrm{C}_{3} \mathrm{C}_{6} \mathrm{H}_{3}$ & 60 & 85 \\
\hline
\end{tabular}

Table $22 \mathrm{ZnO}$ nanoparticles catalyzed synthesis of pyrido[2,3- $d]$ pyrimidine-diones

\section{$\mathrm{CH}_{3} \mathrm{COONH}_{4}$}

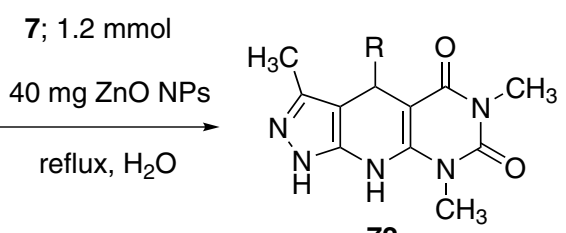

70; $1.1 \mathrm{mmol}$

$$
\text { 17; } 1 \mathrm{mmol}
$$<smiles>[R]C=O</smiles>

67a; $1 \mathrm{mmol}$
5; $1 \mathrm{mmol}$

\begin{tabular}{llllllll}
\hline Entry & $\mathrm{R}$ & Time (h) & Yield (\%) & Entry & $\mathrm{R}$ & Time (h) & Yield (\%) \\
\hline 1 & $\mathrm{C}_{6} \mathrm{H}_{5}$ & 4 & 91 & 6 & $4-\mathrm{NO}_{2} \mathrm{C}_{6} \mathrm{H}_{4}$ & 4 & 91 \\
2 & $4-\mathrm{FC}_{6} \mathrm{H}_{4}$ & 3.5 & 90 & 7 & $3-\mathrm{NO}_{2} \mathrm{C}_{6} \mathrm{H}_{4}$ & 4.2 & 89 \\
3 & $4-\mathrm{ClC}_{6} \mathrm{H}_{4}$ & 3.5 & 91 & 8 & $4-\mathrm{OHC}_{6} \mathrm{H}_{4}$ & 4.5 & 85 \\
4 & $4-\mathrm{BrC}_{6} \mathrm{H}_{4}$ & 3.8 & 90 & 9 & $4-\mathrm{N}_{(}\left(\mathrm{CH}_{3}\right)_{2} \mathrm{C}_{6} \mathrm{H}_{4}$ & 4.5 & 85 \\
5 & $2,4-\mathrm{diClC}_{6} \mathrm{H}_{3}$ & 4 & 90 & & & \\
\hline
\end{tabular}


<smiles>[X]C1=C([R])N([R])C(=O)CC1=O</smiles>

$1 \mathrm{mmol}$<smiles></smiles>

79

6 entries, $91-93 \%$<smiles>[Z]C(=O)c1ccc([R])nc1[Z]</smiles>

80$$
\text { 67aa; } X=O, R^{1}=H
$$$$
\text { 75; } X=S, R^{1}=H
$$

$10 \mathrm{~mol} \% \mathrm{ZnO} N \mathrm{NP}$
neat, $70^{\circ} \mathrm{C}$
$10-56 \mathrm{~min}$<smiles>[Z]C1(C)CC(=O)CC(=O)[Z]1C</smiles>

$1 \mathrm{mmol}$

16; $\mathrm{Z}=\mathrm{CH}_{3}$

43; $Z=O$

$10 \mathrm{~mol} \% \mathrm{ZnO}$ NPs neat, $70{ }^{\circ} \mathrm{C}$ $8-45 \mathrm{~min}$

$$
67 a ; X=O, \mathrm{R}^{1}=\mathrm{CH}_{3}
$$

3 entries, $90-96 \%$<smiles>[R]OC(=O)c1ccc([R])nc1C(=O)O[R16]</smiles><smiles></smiles>

Scheme $10 \mathrm{ZnO}$ nanoparticles catalyzed synthesis of structurally diverse pyridine derivatives

Table $23 \mathrm{ZnO}$ nanoparticles catalyzed synthesis of 1,3-oxazoline-2-thione derivatives

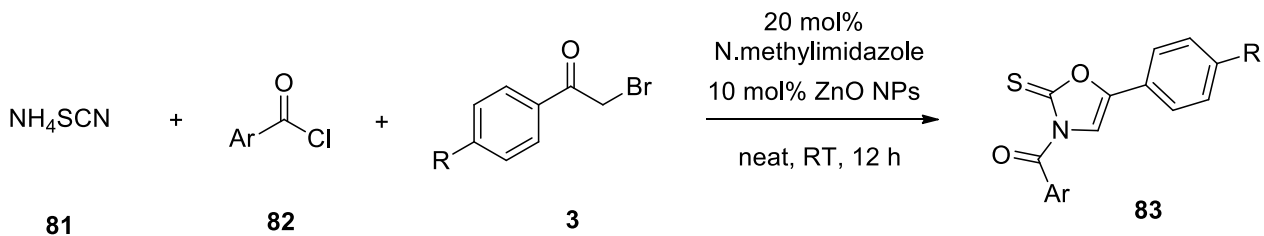

\begin{tabular}{llllllll}
\hline Entry & $\mathrm{Ar}$ & $\mathrm{R}$ & Yield $(\%)$ & Entry & $\mathrm{Ar}$ & $\mathrm{R}$ & Yield (\%) \\
\hline 1 & $4-\mathrm{BrC}_{6} \mathrm{H}_{4}$ & $4-\mathrm{NO}_{2}$ & 95 & 4 & $4-\mathrm{OCH}_{3} \mathrm{C}_{6} \mathrm{H}_{4}$ & $4-\mathrm{Br}$ & 85 \\
2 & $4-\mathrm{NO}_{2} \mathrm{C}_{6} \mathrm{H}_{4}$ & $4-\mathrm{Br}$ & 75 & 5 & $4-\mathrm{BrC}_{6} \mathrm{H}_{4}$ & $4-\mathrm{OCH}_{3}$ & 83 \\
3 & $4-\mathrm{NO}_{2} \mathrm{C}_{6} \mathrm{H}_{4}$ & $4-\mathrm{CH}_{3}$ & 70 & & & \\
\hline
\end{tabular}

\section{Synthesis of pyrano[3,2-b]chromene}

Ziraka et al. [134] prepared bismuth oxide supported nano zinc dioxide [nano $\mathrm{Bi}_{2} \mathrm{O}_{3}-\mathrm{ZnO}$ ] by sol-gel method and characterized it by FT-IR, XRD, SEM, TEM and energy-dispersive X-ray analysis (EDX) analysis. They employed this newly synthesize catalytic system for the synthesis of pyrano[3,2-b]chromenes (87) via one-pot three-component reactions of various aromatic aldehydes (5), kojic acid (86) and dimethone (16) under solvent-free 
Table $24 \mathrm{ZnO}$ nanoparticles catalyzed synthesis of thieno[2,3-c]pyridine derivatives

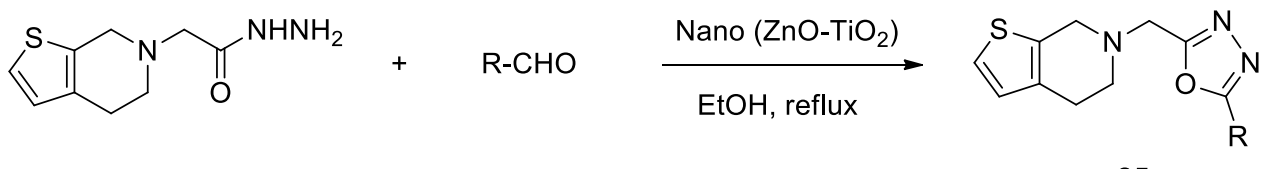

84

5

85

\begin{tabular}{lllll}
\hline Entry & $\mathrm{R}$ & Product & Time (min) & Yield (\%) \\
\hline 1 & $4-\mathrm{ClC}_{6} \mathrm{H}_{4}$ & $\mathbf{8 5 a}$ & 12 & 96 \\
2 & $\mathrm{C}_{6} \mathrm{H}_{5}$ & $\mathbf{8 5 b}$ & 12 & 91 \\
3 & $4-\mathrm{OCH}_{3} \mathrm{C}_{6} \mathrm{H}_{4}$ & $\mathbf{8 5 c}$ & 13 & 95 \\
4 & $3,4-\left(\mathrm{OH}_{2} \mathrm{C}_{6} \mathrm{H}_{3}\right.$ & $\mathbf{8 5 d}$ & 14 & 91 \\
5 & $2-\mathrm{ClC}_{6} \mathrm{H}_{4}$ & $\mathbf{8 5 e}$ & 12 & 95 \\
6 & $2,6-\left(\mathrm{Cl}_{2} \mathrm{C}_{6} \mathrm{H}_{3}\right.$ & $\mathbf{8 5 f}$ & 12 & 91 \\
7 & $2,4-\left(\mathrm{OCH}_{3}\right)_{2} \mathrm{C}_{6} \mathrm{H}_{3}$ & $\mathbf{8 5 g}$ & 14 & 94 \\
8 & $4-\mathrm{OH}_{-}-\mathrm{C}_{6} \mathrm{H}_{4}$ & $\mathbf{8 5 h}$ & 13 & 94 \\
9 & $\mathrm{C}_{4} \mathrm{H}_{4} \mathrm{~N}$ & $\mathbf{8 5 i}$ & 15 & 95 \\
10 & $\mathrm{C}_{4} \mathrm{H}_{3} \mathrm{~S}$ & $\mathbf{8 5 j}$ & 14 & 94 \\
11 & $4-\mathrm{FC}_{6} \mathrm{H}_{4}$ & $\mathbf{8 5 k}$ & 13 & 94 \\
12 & $2,4-\mathrm{diClC}_{6} \mathrm{H}_{3}$ & $\mathbf{8 5 1}$ & 12 & 92 \\
13 & $\mathrm{C}_{5} \mathrm{H}_{4} \mathrm{~N}$ & $\mathbf{8 5 m}$ & 15 & 95 \\
\hline
\end{tabular}

Table $25 \mathrm{ZnO}$ nanoparticles catalyzed synthesis of pyrano[3,2-b]chromene<smiles>[R]C(=O)O[13C](=O)[O-]</smiles>

\begin{tabular}{llllll}
\hline Entry & $\mathrm{R}$ & Yield (\%) & Entry & $\mathrm{R}$ & Yield (\%) \\
\hline 1 & $\mathrm{C}_{6} \mathrm{H}_{5}$ & 80 & 6 & $4-\mathrm{NO}_{2} \mathrm{C}_{6} \mathrm{H}_{4}$ \\
2 & $4-\mathrm{ClC}_{6} \mathrm{H}_{4}$ & 82 & 7 & $2,4-\mathrm{diClC}_{6} \mathrm{H}_{3}$ \\
3 & $2-\mathrm{ClC}_{6} \mathrm{H}_{4}$ & 79 & 8 & $4-\mathrm{OCH}_{3} \mathrm{C}_{6} \mathrm{H}_{4}$ & 78 \\
4 & $3-\mathrm{NO}_{2} \mathrm{C}_{6} \mathrm{H}_{4}$ & 84 & 9 & $4-\mathrm{CH}_{3} \mathrm{C}_{6} \mathrm{H}_{4}$ & 75 \\
\hline
\end{tabular}

condition at $100{ }^{\circ} \mathrm{C}$ (Table 25). During optimization it was found that the catalytic activity of combined nano $\left(\mathrm{ZnO}-\mathrm{TiO}_{2}\right)$ is superior to the individual effect of nano $\mathrm{ZnO}$ or nano $\mathrm{Bi}_{2} \mathrm{O}_{3}$.

\section{Synthesis of 1,2,3-triazoles}

Albadi et al. [135] synthesized $\mathrm{CuO}$ supported nano-ZnO [nano-CuO-ZnO] by a co-precipitation method and well characterized it by XRD, SEM, TEM and EDS analysis.
Using this newly prepared efficient catalytic system they synthesized a series of 1,2,3-triazoles (89) via one-pot three-component reactions of various benzyl halides $(\mathbf{8 8})$ phenylacetylenes (48) and sodium azide (41) in water under reflux condition (Table 26). During optimization combined nano $(\mathrm{CuO}-\mathrm{ZnO})$ showed better catalytic efficacy than the individual nano- $\mathrm{ZnO}$ or nano-CuO. After completion of reaction, the catalyst was recovered easily and recycled for several runs without loss in catalytic activity. 
Table $26 \mathrm{ZnO}$ nanoparticles catalyzed synthesis of 1,2,3-triazoles
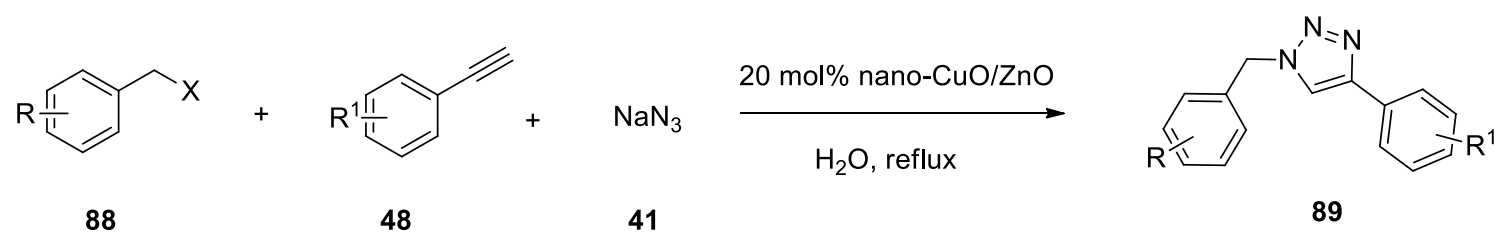

\begin{tabular}{llllll}
\hline Entry & $\mathrm{R}$ & $\mathrm{X}$ & $\mathrm{R}^{1}$ & Time (min) & Yield (\%) \\
\hline 1 & $\mathrm{H}$ & $\mathrm{Br}$ & $\mathrm{H}$ & 20 & 92 \\
2 & $3-\mathrm{CH}_{3}$ & $\mathrm{Br}$ & $\mathrm{H}$ & 20 & 91 \\
3 & $4-\mathrm{OCH}_{3}$ & $\mathrm{Br}$ & $\mathrm{H}$ & $\mathrm{H}$ & 92 \\
4 & $2,4-\mathrm{diCl}$ & $\mathrm{Br}$ & $\mathrm{H}$ & 35 & 90 \\
5 & $4-\mathrm{NO}_{2}$ & $\mathrm{Br}$ & $3-\mathrm{NH}_{2}$ & 30 & 89 \\
6 & $\mathrm{H}$ & $\mathrm{Br}$ & $3-\mathrm{NH}_{2}$ & 20 & 90 \\
7 & $4-\mathrm{OCH}_{3}$ & $\mathrm{Br}$ & $\mathrm{H}$ & 30 & 92 \\
8 & $\mathrm{H}$ & $\mathrm{Cl}$ & $\mathrm{H}$ & 18 & 89 \\
9 & $2,4-\mathrm{diCl}$ & $\mathrm{Cl}$ & & & 9 \\
\hline
\end{tabular}

\section{Conclusions}

The present review describes the recent developments on the synthesis of biologically promising heterocycles using nano zinc oxide as a mild, cheap, non-toxic, efficient, reusable, Lewis acidic heterogeneous catalyst. For many organic transformations, catalytic efficacy of nano $\mathrm{ZnO}$ was found to be superior to the commercially available bulk $\mathrm{ZnO}$. After completion of reaction, in many occasions nano-ZnO was successfully recovered and reused further for the several runs without significant loss in catalytic activity. This review will enrich the readers about the developments of $\mathrm{ZnO}$ nanoparticles catalyzed synthesis of various heterocycles reported so far. Therefore, the present review will surely attract attention of the organic methodologists working with this fascinating catalyst worldwide.

Acknowledgements The author is grateful to Dr. Sudhir Kartha, Chancellor, Indus International University, Una, Himachal Pradesh, India for his wholehearted support throughout and the Kartha Education Society, Mumbai, India for the financial help. Special thanks are due to Mr. Pankaj Choudhary, Dr. Samik Mukherjee and Dr. Sudipta Som for their able assistance to search the literature.

Open Access This article is distributed under the terms of the Creative Commons Attribution 4.0 International License (http:// creativecommons.org/licenses/by/4.0/), which permits unrestricted use, distribution, and reproduction in any medium, provided you give appropriate credit to the original author(s) and the source, provide a link to the Creative Commons license, and indicate if changes were made.

\section{References}

1. Brahmachari, G.: Handbook of pharmaceutical natural products, 1st edn. Wiley-VCH, Weinheim (2010)

2. Banerjee, B.: Recent developments on ultrasound-assisted onepot multicomponent synthesis of biologically relevant heterocycles. Ultrason. Sonochem. 35, 15-35 (2017)

3. Wu, J.Y., Fong, W.F., Zhang, J.X., Leung, C.H., Kwong, H.L., Yang, M.S., Li, D., Cheung, H.Y.: Reversal of multidrug resistance in cancer cells by pyranocoumarins isolated from Radix Peucedani. Eur. J. Pharmacol. 473, 9-17 (2003)

4. Raj, T., Bhatia, R.K., Kapur, A., Sharma, M., Saxena, A.K., Ishar, M.P.S.: Cytotoxic activity of 3-(5-phenyl-3H-[1, 2, 4] dithiazol-3-yl)chromen-4-ones and 4-oxo-4H-chromene-3-carbothioic acid $N$-phenylamides. Eur. J. Med. Chem. 45, 790-794 (2010)

5. De Andrade-Neto, V.F., Goulart, M.O., Da Silva Filho, J.F., Da Silva, M.J., Pinto, M.D.C., Pinto, A.V., Zalis, M.G., Carvalho, L.H., Krettli, A.U.: Antimalarial activity of phenazines from lapachol, beta-lapachone and its derivatives against Plasmodium falciparum in vitro and Plasmodium berghei in vivo. Bioorg. Med. Chem. Lett. 14, 1145-1149 (2004)

6. Foye, W.O.: Principi di Chemico Farmaceutica. Piccin, Padora (1991)

7. Moon, D.O., Kim, K.C., Jin, C.Y., Han, M.H., Park, C., Lee, K.J., Park, Y.M., Choi, Y.H., Kim, G.Y.: Inhibitory effects of eicosapentaenoic acid on lipopolysaccharide-induced activation in BV2 microglia. Int. Immunopharmacol. 7, 222-229 (2007)

8. Rueping, M., Sugiono, E., Merino, E.: Asymmetric organocatalysis: an efficient enantioselective access to benzopyranes and chromenes. Chem. Eur. J. 14, 6329-6332 (2008)

9. Banerjee, B.: Bismuth(III) triflate: an efficient catalyst for the synthesis of diverse biologically relevant heterocycles. Chem. Select 2, 6744-6757 (2017)

10. Banerjee, B.: [Bmim] $\mathrm{BF}_{4}$ : a versatile ionic liquid for the synthesis of diverse bioactive heterocycles. Chem. Select 2, 8326-8376 (2017)

11. Thatipalli, P., Kumar, R., Bulusu, C., Chakka, R., Padi, P.R., Yerra, A., Bollikonda, S.: Synthesis and characterization of 
impurities of an anti-psychotic drug substance. Olanzapine. Arkivoc 11, 195-201 (2008)

12. Liao, Y., DeBoer, P., Meier, E., Wikstrm, H.: Synthesis and pharmacological evaluation of triflate-substituted analogues of Clozapine: identification of a novel atypical neuroleptic. J. Med. Chem. 40, 4146-4153 (1997)

13. Barbosa, L.C.A., Maltha, C.R.A., Lage, M.R., Barcelos, R.C., Dona, A., Carneiro, J.W.M., Forlani, G.: Synthesis of Rubrolide analogues as new inhibitors of the photosynthetic electron transport chain. J. Agric. Food Chem. 60, 10555-10563 (2012)

14. Chernyak, N., Gevorgyan, V.: General and efficient copper-catalyzed three-component coupling reaction towards imidazoheterocycles: one-pot synthesis of Alpidem and Zolpidem. Angew. Chem. Int. Ed. 49, 2743-2746 (2010)

15. Bagdi, A.K., Rahman, M., Santra, S., Majee, A., Hajra, A.: Copper-catalyzed synthesis of imidazo[1,2-a]pyridines through tandem imine formation-oxidative cyclization under ambient air: one-step synthesis of Zolimidine on a gram-scale. Adv. Synth. Catal. 355, 1741-1747 (2013)

16. Hinkle, J.L., Cheever, K.H.: Brunner \& Suddarth's textbook of medical-surgical nursing, 13th edn. Wolters Kluwer Health/Lippincott Williams \& Wilkins, Philadelphia (2014)

17. Banerjee, B.: Recent developments on ultrasound assisted catalyst-free organic synthesis. Ultrason. Sonochem. 35, 1-14 (2017)

18. Banerjee, B.: $\mathrm{Sc}(\mathrm{OTf})_{3}$ catalyzed carbon-carbon and carbonheteroatom bond forming reactions: A review. Arkivoc 1, 1-25 (2017)

19. Chaturvedi, S., Dave, P.N., Shah, N.K.: Applications of nanocatalyst in new era. J. Saudi Chem. Soc. 16, 307-325 (2012)

20. Pla, D., Gómez, M.: Metal and metal oxide nanoparticles: a lever for C-H functionalization. ACS Catal. 6, 3537-3552 (2016)

21. Saha, P., Ramana, T., Purkait, N., Ali, M.A., Paul, R., Punniyamurthy, T.: Ligand-free copper-catalyzed synthesis of substituted benzimidazoles, 2-aminobenzimidazoles, 2-aminobenzothiazoles, and benzoxazoles. J. Org. Chem. 74, 8719-8725 (2009)

22. Ahmadi, S.J., Sadjadi, S., Hosseinpour, M., Outokesh, M., Hekmatshoar, R.: A heterogeneous strong basic nanocrystalline copper(II) oxide catalyst for efficient synthesis of 4-keto4,5,6,7-tetrahydrobenzofurans. Catal. Commun. 10, 1423-1426 (2009)

23. Agawane, S.M., Nagarkar, J.M.: Nano ceria catalyzed synthesis of $\alpha$-aminophosphonates under ultrasonication. Tetrahedron Lett. 52, 3499-3504 (2011)

24. Zhang, J., Cheng, P., Ma, Y., Liu, J., Miao, Z., Ren, D., Fan, C., Liang, M., Liu, L.: An efficient nano CuO-catalyzed synthesis and biological evaluation of quinazolinone Schiff base derivatives and bis-2,3-dihydroquinazolin-4(1H)-ones as potent antibacterial agents against Streptococcus lactis. Tetrahedron Lett. 57, 5271-5277 (2016)

25. Huang, L., Liu, W., Wu, J., Fu, Y., Wang, K., Huo, C., Du, Z.: Nano-copper catalyzed three-component reaction to construct 1,4-substituted 1,2,3-triazoles. Tetrahedron Lett. 55, 2312-2316 (2014)

26. Ghasemzadeh, M.A., Safaei-Ghomi, J., Zahedi, S.: $\mathrm{Fe}_{3} \mathrm{O}_{4}$ nanoparticles: a highly efficient and easily reusable catalyst for the one-pot synthesis of xanthene derivatives under solvent-free conditions. J. Serb. Chem. Soc. 78, 769-779 (2013)

27. Mollashahi, E., Nikraftar, M.: Nano-SiO2 catalyzed threecomponent preparations of pyrano[2,3-d]pyrimidines, 4H-chromenes, and dihydropyrano[3,2-c]chromenes. J. Saudi Chem. Soc. (2017). https://doi.org/10.1016/j.jscs.2017.06.003

28. Safaei-Ghomi, J., Ghasemzadeha, M.A., Mehrabi, M.: Calcium oxide nanoparticles catalyzed one-step multicomponent synthesis of highly substituted pyridines in aqueous ethanol media. Sci. Iran. C. 20, 549-554 (2013)
29. Edayadulla, N., Lee, Y.R.: Cerium oxide nanoparticle-catalyzed three-component protocol for the synthesis of highly substituted novel quinoxalin-2-amine derivatives and 3,4-dihydroquinoxalin-2-amines in water. RSC Adv. 4, 11459-11468 (2014)

30. Santra, S., Rahman, M., Roy, A., Majee, A., Hajra, A.: Nanoindium oxide: an efficient catalyst for one-pot synthesis of 2,3-dihydroquinazolin-4(1H)-ones with a greener prospect. Catal. Commun. 49, 52-57 (2014)

31. Bajpai, S., Singh, S., Srivastava, V.: Nano zirconia catalysed one-pot synthesis of some novel substituted imidazoles under solvent-free conditions. RSC Adv. 5, 28163-28170 (2015)

32. Chandra, D., Mridha, S., Basak, D., Bhaumik, A.: Template directed synthesis of mesoporous $\mathrm{ZnO}$ having high porosity and enhanced optoelectronic properties. Chem. Commun. 17, 2384-2386 (2009)

33. Djurísíc, A.B., Ng, A.M.C., Chen, X.Y.: ZnO nanostructures for optoelectronics: material properties and device applications. Prog. Quant. Electron. 34, 191-259 (2010)

34. Wan, W., Huang, J., Zhu, L., Hu, L., Wen, Zh, Sun, L., Ye, Zh: Defects induced ferromagnetism in $\mathrm{ZnO}$ nanowire arrays doped with copper. Cryst. Eng. Comm. 15, 7887-7894 (2013)

35. Zhai, T., Fang, X., Liao, M., Xu, X., Zeng, H., Yoshio, B., Golberg, D.: A comprehensive review of one-dimensional metaloxide nanostructure photodetectors. Sensors 9, 6504-6529 (2009)

36. Bhakat, C., Singh, P.P.: Zinc oxide nanorods: synthesis and its applications in solar cell. IJMER 2, 2452-2454 (2012)

37. Gupta, S.K., Joshi, A., Kaur, M.: Development of gas sensors using ZnO nanostructures. J. Chem. Sci. 122, 57-62 (2010)

38. Hossain, M.K., Ghosh, S.C., Boontongkong, Y., Thanachayanont, C., Dutta, J.: Growth of zinc oxide nanowires and nanobelts for gas sensing applications. J. Metastab. Nanocryst. Mater. 23, 27-30 (2005)

39. Singh, G., Joyce, E.M., Beddow, J., Mason, T.J.: Evaluation of antibacterial activity of $\mathrm{ZnO}$ nanoparticles coated sonochemically on to textile fabrics. J. Microbiol. Biotechnol. Food Sci. 2, 106-120 (2012)

40. Das, D., Nath, B.C., Phukon, P., Kalita, A., Dolui, S.K.: Synthesis of $\mathrm{ZnO}$ nanoparticles and evaluation of antioxidant and cytotoxic activity. Colloids Surf. B 111, 556-560 (2013)

41. Lao, J.Y., Huang, J.Y., Wang, D.Z., Ren, Z.F.: ZnO nanobridges and nanonails. Nano Lett. 3, 235-238 (2003)

42. Öztürk, S., Kilinc, N., Tasaltin, N., Öztürk, Z.Z.: Fabrication of $\mathrm{ZnO}$ nanowires and nanorods. Physica E 44, 1062-1065 (2012)

43. Gao, P.X., Ding, Y., Wang, Z.L.: Electronic transport in superlattice-structured $\mathrm{ZnO}$ nanohelix. Nano Lett. 9, 137-143 (2009)

44. Hasnidawani, J.N., Azlina, H.N., Norita, H., Bonnia, N.N., Ratim, S., Ali, E.S.: Synthesis of $\mathrm{ZnO}$ nanostructures using sol-gel method. Procedia Chem. 19, 211-216 (2016)

45. Hsieh, C.-H.: Spherical zinc oxide nano particles from zinc acetate in the precipitation method. J. Chin. Chem. Soc. 54, 31-34 (2007)

46. Ahmed, S., Chaudhry, S.A., Ikram, S.: A review on biogenic synthesis of $\mathrm{ZnO}$ nanoparticles using plant extracts and microbes: A prospect towards green chemistry. J. Photochem. Photobio. B Biol. 166, 272-284 (2017)

47. MaGee, D.I., Dabiri, M., Salehi, P., Torkian, L.: Highly efficient one-pot three-component Mannich reaction catalyzed by ZnO-nanoparticles in water. Arkivoc 11, 156-164 (2011)

48. Hosseini-Sarvari, M., Sharghi, H., Etemad, S.: Nanocrystalline $\mathrm{ZnO}$ for Knoevenagel condensation and reduction of the carbon, carbon double bond in conjugated alkenes. Helv. Chim. Acta 91, 715-724 (2008)

49. Sandhu, J.S.: ZnO nanobelts: an efficient catalyst for synthesis of 5-arylidine-2,4-thiazolidinediones and 5-arylidine-Rhodanines. Int. J. Org. Chem. 2, 305-310 (2012) 
50. Gharib, A., Pesyan, N.N., Fard, L.V., Roshani, M.: ZnO nanoparticle as catalyst for efficient green synthesis of antiplatelet drug (Clopidogrel). J. Chem. Eng. Chem. Res. 1, 1-5 (2014)

51. Hosseini-Sarvari, M., Etemad, S.: Nanosized zinc oxide as a cata-lyst for the rapid and green synthesis of $\beta$-phosphono malonates. Tetrahedron 64, 5519-5523 (2003)

52. Hosseini-Sarvari, M.: An efficient and eco-friendly nanocrystalline zinc oxide catalyst for one-pot, three component synthesis of new ferrocenyl aminophosphonic esters under solventfree condition. Catal. Lett. 141, 347-355 (2011)

53. Hosseini-Sarvari, M., Tavakolian, M.: Preparation, characteriza-tion, and catalysis application of nano-rods zinc oxide in the synthesis of 3-indolyl-3-hydroxy oxindoles in water. Appl. Catal. A: Gen. 441-442, 65-71 (2012)

54. Mirjafary, Z., Saeidian, H., Sadeghi, A., Moghaddam, F.M.: $\mathrm{ZnO}$ nanoparticles: an efficient nanocatalyst for the synthesis of $\beta$-acetamido ketones/esters via a multi-component reaction. Catal. Commun. 9, 299-306 (2008)

55. Moghaddam, F.M., Saeidian, H.: Controlled microwaveassisted synthesis of $\mathrm{ZnO}$ nanopowder and its catalytic activity for $O$-acylation of alcohol and phenol. Mater. Sci. Eng. B Environ. 139, 265-269 (2007)

56. Indulkar, U.U., Kale, S.R., Gawande, M.B., Jayaram, R.V.: Ecofriendly and facile Nano $\mathrm{ZnO}$ catalyzed solvent-free enamination of 1,3-dicarbonyls. Tetrahedron Lett. 53, 3857-3860 (2012)

57. Gharib, A., Pesyan, N.N., Vojdanifard, L., Jahangir, M., Roshani, M., Moghadasi, S.: Synthesis of $\beta$-amino carbonyl compounds using $\mathrm{ZnO}$ nanoparticles as a green, effective and reusable catalyst. Bul. Chem. Commun. 46, 486-496 (2014)

58. Di Santo, R., Costi, R., Artico, M., Miele, G., Lavecchia, A., Novellino, E., Bergamini, A., Cancio, R., Maga, G.: Arylthiopyrrole (AThP) derivatives as non-nucleoside HIV-1 reverse transcriptase inhibitors: synthesis, structure-activity relationships, and docking studies (Part 1). ChemMedChem 1, 1367-1378 (2006)

59. Zeng, L., Miller, E.W., Pralle, A., Isacoff, E.Y., Chang, C.J.: A selective turn-on fluorescent sensor for imaging copper in living cells. J. Am. Chem. Soc. 128, 10-11 (2006)

60. Clark, B.R., Capon, R.J., Lacey, E., Tennant, S., Gill, J.H.: Polyenylpyrroles and polyenylfurans from an australian isolate of the soil Ascomycete Gymnoascus reessii. Org. Lett. 8, 701-704 (2006)

61. La Regina, G., Silvestri, R., Artico, M., Lavecchia, A., Novellino, E., Befani, O., Turini, P., Agostinelli, E.: New pyrrole inhibitors of monoamine oxidase: synthesis, biological evaluation, and structural determinants of MAO-A and MAO-B selectivity. J. Med. Chem. 50, 922-931 (2007)

62. Rossi, R., Montecucco, A., Capolongo, L., Mezzina, M., Chevallier-Lagente, O., Sarasin, A., Ciarrocchi, G.: The alkylating antitumor drug tallimustine does not induce DNA repair. Anticancer Res. 16, 3779-3783 (1996)

63. Zhang, T.: Physiologically based pharmacokinetic modeling of disposition and drug-drug interactions for atorvastatin and its metabolites. Eur. J. Pharm. Sci. 77, 216-229 (2015)

64. Sabbaghan, M., Ghalaei, A.: Catalyst application of $\mathrm{ZnO}$ nanostructures in solvent free synthesis of polysubstituted pyrroles. J. Mol. Liq. 193, 116-122 (2014)

65. Lombardino, J.G., Wiseman, E.H.: Preparation and anti-inflammatory activity of some nonacidic trisubstituted imidazoles. J. Med. Chem. 17, 1182-1188 (1974)

66. Wang, L., Woods, K.W., Li, Q., Barr, K.J., McCroskey, R.W., Hannick, S.M., Gherke, L., Credo, R.B., Hui, Y.H., Marsh, K., Warner, R., Lee, J.Y., Mozng, N.Z., Frost, D., Rosenberg, S.H., Sham, H.L.: Potent, orally active heterocycle-based combretastatin A-4 analogues: synthesis, structure-activity relationship, pharmaco kinetics, and in vivo antitumor activity evaluation. J. Med. Chem. 45, 1697-1711 (2002)

67. Pozherskii, A.F., Soldatenkov, A.T., Katritzky, A.Y.: Heterocycles in life and society. Wiley, New York (1997)

68. Lindberg, P., Nordberg, P., Alminger, T., Brandstorm, A., Wallmark, B.: The mechanism of action of the antisecretory agent omeprazole. J. Med. Chem. 29, 1327-1329 (1986)

69. Nikoofar, K., Haghighi, M., Lashanizadegan, M., Ahmadvand, $\mathrm{Z}$.: $\mathrm{ZnO}$ nanorods: efficient and reusable catalysts for the synthesis ofsubstituted imidazoles in water. J. Taibah Univ. Sci. 9, 570-578 (2015)

70. Alinezhad, H., Salehian, F., Biparva, P.: Synthesis of benzimidazole derivatives using heterogeneous zno nanoparticles. Synth. Commun. 42, 102-108 (2012)

71. Paul, B., Vadivel, S., Dhar, S.S., Debbarma, S., Kumaravel, M.: One-pot green synthesis of zinc oxide nano rice and its application as sonocatalyst for degradation of organic dye and synthesis of 2-benzimidazole derivatives. J. Phy. Chem. Solid 104, 152-159 (2017)

72. Swami, S., Devi, N., Agarwala, A., Singh, V., Shrivastava, $\mathrm{R} .: \mathrm{ZnO}$ nanoparticles as reusable heterogeneous catalyst for efficient one pot three component synthesis of imidazo-fused polyheterocycles. Tetrahedron Lett. 57, 1346-1350 (2016)

73. Kassaee, M.Z., Masrouri, H., Movahedi, F.: ZnO-nanoparticlepromoted synthesis of polyhydroquinoline derivatives via multicomponent Hantzsch reaction. Monatsh. Chem. 141, 317-322 (2010)

74. Reen, G.K., Ahuja, M., Kumar, A., Patidar, R., Sharma, P.: $\mathrm{ZnO}$ Nanoparticle-catalyzed multicomponent reaction for the synthesis of 1,4-diaryl dihydropyridines. Org. Prep. Proced. Int. 49, 273-286 (2017)

75. Shafiee, M.R.M., Moloudi, R., Ghashang, M.: ZnO nanopowder: an efficient catalyst for the preparation of 2,4,6-triaryl pyridines under solvent-free condition. APCBEE Procedia 1, 221-225 (2012)

76. Sadeghi, B., Karimi, F.: ZnO nanoparticlesas an efficient and reusable catalyst for synthesis of quinoxalineunder solvent free condition. Iran. J. Catal. 3, 1-7 (2013)

77. Kappe, C.O.: 100 years of the Biginelli dihydropyrimidine synthesis. Tetrahedron 49, 6937-6963 (1993)

78. Biginelli, P., Gazz, P.: Synthesis of 3,4-Dihydropyrimidin-2(1H)Ones. Chim. Ital. 23, 360 (1893)

79. Hassanpour, A., Khanmiri, R.H., Abolhasani, J.: ZnO nanoparticles as an efficient, heterogeneous, reusable, and eco-friendly catalyst for one-pot three-component synthesis of 3,4-dihydropyrimidin-2(1H)-(thio)one derivatives in water. Synth. Commun. 45, 727-733 (2015)

80. Abdolmohammadi, S.: ZnO-nanoparticles-catalyzed cyclocondensation reaction of arylmethylidenepyruvic acids with 6-aminouracils. Comb. Chem. High Throughput Screen. 16, 32-36 (2013)

81. Azarifar, A., Nejat-Yami, R., Azarifar, D.: Nano-ZnO: an efficient and reusable catalyst for one-pot synthesis of $1 H$-pyrazolo[1,2$b]$ phthalazine-5,10-diones and pyrazolo[1,2-a][1, 2, 4]triazole1,3-diones. J. Iran. Chem. Soc. 10, 297-306 (2013)

82. Ager, I.R., Barnes, A.C., Danswan, G.W., Hairsine, P.W., Kay, D.P., Kennewell, P.D., Matharu, S.S., Miller, P., Robson, P., Rowlands, D.A., Tully, W.R., Westwood, R.: Synthesis and oral antiallergic activity of carboxylic acids derived from imidazo[2,1-c][1, 4]benzoxazines, imidazo[1,2-a]quinolines, imidazo[1,2-a]quinoxalines, imidazo[1,2-a]quinoxalinones, pyrrolo[1,2-a]quinoxalinones, pyrrolo[2,3-a]quinoxalinones, and imidazo[2,1- $b$ ]benzothiazoles. J. Med. Chem. 31, 1098-1115 (1988)

83. Clements, S.J., Danswan, G., Gardner, C.R., Matharu, S.S., Murdoch, R., Tully, W.R., Westwood, R.: (Imidazo[1,2-a] 
pyrimidin-2-yl)phenylmethanones and related compounds as potential nonsedative anxiolytics. J. Med. Chem. 31, 1220-1226 (1988)

84. Abaszadeh, M., Seifi, M., Asadipour, A.: Ultrasound promotes one-pot synthesis of 1,4-dihydropyridine and imidazo[1,2-a]quinoline derivatives, catalyzed by $\mathrm{ZnO}$ nanoparticles. Res. Chem. Intermed. 41, 5229-5238 (2015)

85. Shaabani, A., Maleki, A.: Ionic liquid promoted one-pot threecomponent reaction: synthesis of annulated imidazo[1,2-a]azines using trimethylsilylcyanide. Monatsh. Chem. 138, 51-56 (2007)

86. Sun, C., Ji, S.J., Liu, Y.: A novel, simple and efficient synthesis of 3-amino-benzo[ $[d]$ imidazo[2,1- $b]$ thiazole derivatives via a multicomponent procedure. J. Chin. Chem. Soc. 55, 292-296 (2008)

87. Rupert, K.C., Henry, J.R., Dodd, J.H., Wadsworth, S.A., Cavender, D.E., Olini, G.C., Fahmy, B., Siekierka, J.: Imidazopyrimidines, potent inhibitors of p38 MAP kinase. Bioorg. Med. Chem. Lett. 13, 347-350 (2003)

88. Katritzky, A.R., Xu, Y.J., Tu, H.: Regiospecific synthesis of 3 -substituted imidazo[1,2-a]pyridines, imidazo[1,2-a]pyrimidines, and imidazo[1,2-c]pyrimidine. J. Org. Chem. 68, 49354937 (2003)

89. Sadjadi, S., Eskandari, M.: Ultrasonic assisted synthesis of imidazo[1,2- $a$ ]azine catalyzed by $\mathrm{ZnO}$ nanorods. Ultrason. Sonochem. 20, 640-643 (2013)

90. Kantam, M.L., Kumar, K.B.S., Sridhar, C.: Nanocrystalline ZnO as an efficient heterogeneous catalyst for the synthesis of 5-substituted $1 H$-tetrazoles. Adv. Synth. Catal. 347, 1212-1214 (2005)

91. Leyva-Perez, A., Cabrero-Antonino, J.R., Corma, A.: Bifunctional solid catalysts for chemoselective hydrogenation-cyclisation-amination cascade reactions of relevance for the synthesis of pharmaceuticals. Tetrahedron 66, 8203-8209 (2010)

92. Kruse, H.: Clobazam: induction of hyperlocomotion in a new nonautomatized device for measuring motor activity and exploratory behavior in mice: Comparison with diazepam and critical evaluation of the results with an automatized hole-board apparatus ("Planche a Trous"). Drug Dev. Res. 2, 145-151 (1982)

93. Ghasemzadeh, M.A., Safaei-Ghomi, J.: Synthesis and characterization of $\mathrm{ZnO}$ nanoparticles: application to one-pot synthesis of benzo[b][1,5]diazepines. Cogent Chem. 1, 1095060 (2015)

94. Miao, S., Andersen, R.J.: Rubrolides A-H, metabolites of the colonial tunicate Ritterella rubra. J. Org. Chem. 56, 6275-6280 (1991)

95. Kotora, M., Negishi, E.: Highly efficient and selective procedures for the synthesis of $\gamma$-alkylidenebutenolides via palladiumcatalyzed ene-yne coupling and palladium- or silver catalyzed lactonization of (Z)-2-en-4-ynoic acids. Synthesis of Rubrolides A, C, D, and E. Synthesis 1, 121-128 (1997)

96. Pour, M., Spulak, M., Buchta, V., Kubanova, P., Voprsalova, M., Wsol, V., Fakova, H., Koudelka, P., Pourova, H.: 3-Phenyl5-acyloxymethyl-2H,5H-furan-2-ones: synthesis and biological activity of a novel group of potential antifungal drugs. J. Med. Chem. 44, 2701-2706 (2001)

97. Lattmann, E., Sattayasai, N., Schwalbe, C.S., Niamsanit, S., Billington, D.C., Lattmann, P., Langley, C.A., Singh, H., Dunn, S.: Novel anti-bacterials against MRSA: synthesis of focussed combinatorial libraries of tri-substituted $2(5 H)$-furanones. Curr. Drug Discov. Technol. 3, 125-134 (2006)

98. Weber, V., Coudert, P., Rubat, C., Duroux, E., Vallee-Goyet, D., Gardette, D., Bria, M., Albuisson, E., Leal, F., Gramain, J.C., Couquelet, J., Madesclaire, M.: Novel 4,5-diaryl-3-hydroxy$2(5 H)$-furanones as anti-oxidants and anti-inflammatory agents. Bioorg. Med. Chem. 10, 1647 (2002)

99. El-Tombary, A., Abdel-Ghany, Y., Belal, A., Shams, E.-D., Soliman, F.: Synthesis of some substituted furan-2(5H)-ones and derived quinoxalinones as potential anti-microbial and anticancer agents. Med. Chem. Res. 20, 865-876 (2011)
100. Lattmann, E., Ayuko, W.O., Kinchinaton, D., Langley, C.A., Singh, H., Karimi, L., Tisdale, M.J.: Synthesis and evaluation of 5-arylated $2(5 \mathrm{H})$-furanones and 2-arylated pyridazin-3 $(2 \mathrm{H})$ ones as anti-cancer agents. J. Pharm. Pharmacol. 55, 1259-1265 (2003)

101. Gundogdu-Karaburun, N., Benkli, K., Tunali, Y., Ucucu, U.: Synthesis and antifungal activities of some aryl [3-(imidazol1-yl/triazol-1-ylmethyl) benzofuran-2-yl] ketoximes. Eur. J. Med. Chem. 41, 651-656 (2006)

102. Baraldi, P.G., Romagnoli, R., Beria, I., Cozzi, P., Geroni, C., Mongelli, N., Bianchi, N., Mischiati, C., Gambari, R.: Synthesis and antitumor activity of new benzoheterocyclic derivatives of distamycin A. J. Med. Chem. 43, 2675-2684 (2000)

103. Tekale, S.U., Kauthale, S.S., Pagore, V.P., Jadhav, V.B., Pawar, R.P.: ZnO nanoparticle-catalyzed efficient one-pot three-component synthesis of 3,4,5-trisubstituted furan-2(5H)-ones. J. Iran. Chem. Soc. 10, 1271-1277 (2013)

104. Safaei-Ghomi, J., Ghasemzadeh, M.A.: Zinc oxide nanoparticle promoted highly efficient one pot three-component synthesis of 2,3-disubstituted benzofurans. Arab. J. Chem. 10, S1774-S1780 (2017)

105. Brahmachari, G., Banerjee, B.: Facile and one-pot access to diverse and densely functionalized 2-amino-3-cyano- $4 H$-pyrans and pyran-annulated heterocyclic scaffolds via an eco-friendly multicomponent reaction at room temperature using urea as a novel organo-catalyst. ACS Sustain. Chem. Eng. 2, 411-422 (2014)

106. Brahmachari, G., Banerjee, B.: Facile and chemically sustainable one-pot synthesis of a wide array of fused $O$ - and $N$-heterocycles catalyzed by trisodium citrate dihydrate under ambient conditions. Asian J. Org. Chem. 5, 271-286 (2016)

107. Brahmachari, G., Laskar, S., Banerjee, B.: Eco-friendly, one-pot multicomponent synthesis of pyran annulated heterocyclic scaffolds at room temperature using ammonium or sodium formate as non-toxic catalyst. J. Heterocycl. Chem. 51, E303-E308 (2014)

108. Bhattacharyya, P., Pradhan, K., Paul, S., Das, A.R.: Nano crystalline $\mathrm{ZnO}$ catalyzed one pot multicomponent reaction for an easy access of fully decorated $4 \mathrm{H}$-pyran scaffolds and its rearrangement to 2-pyridone nucleus in aqueous media. Tetrahedron Lett. 53, 4687-4691 (2012)

109. Zavar, S.: A novel three component synthesis of 2-amino- $4 H-$ chromenes derivatives using nano $\mathrm{ZnO}$ catalyst. Arab. J. Chem. 10, S67-S70 (2017)

110. Mulakayala, N., Murthy, P.V.N.S., Rambabu, D., Aeluri, M., Adepu, R., Krishna, G.R., Reddy, C.M., Prasad, K.R.S., Chaitanya, M., Kumar, C.S., Rao, M.V.B., Pal, M.: Catalysis by molecular iodine: a rapid synthesis of 1,8-dioxo-octahydroxanthenes and their evaluation as potential anticancer agents. Bioorg. Med. Chem. Lett. 22, 2186-2191 (2012)

111. Nakhi, A., Rahman, M.S., Archana, S., Kishore, R., Seerapu, G.P.K., Kumar, K.L., Haldar, D., Pal, M.: Construction and functionalization of pyranone ring fused with pyran moiety: design and synthesis of novel pyrano[4,3- $b]$ pyran-5(4H)-ones as potential inhibitors of sirtuins. Bioorg. Med. Chem. Lett. 23, 4195-4205 (2013)

112. Banerjee, B., Brahmachari, G.: Ammonium chloride catalysed one-pot multicomponent synthesis of 1,8-dioxo-octahydroxanthenes and $N$-aryl-1,8-dioxodecahydroacridines under solvent free conditions. J. Chem. Res. 38, 745-750 (2014)

113. Safaei-Ghomi, J., Ghasemzadeh, M.A., Zahedi, S.: ZnO Nanoparticles: a highly effective and readily recyclable catalyst for the one-pot synthesis of 1,8-dioxo-decahydroacridine and 1,8-dioxooctahydro-xanthene derivatives. J. Mex. Chem. Soc. 57, 1-7 (2013)

114. Safaei-Ghomi, J., Ghasemzadeh, M.A.: A simple and efficient synthesis of 12-aryl-8,9,10,12-tetrahydrobenzo[a] 
xanthen-11-ones by $\mathrm{ZnO}$ nanoparticles catalyzed three component coupling reaction of aldehydes, 2-naphthol and dimedone. S. Afr. J. Chem. 67, 27-32 (2014)

115. Piltan, M.J., Safaei-Ghomi, J.: Nano crystalline Zno catalyzed one pot three-component synthesis of 7-alkyl-6H,7 H-naphtho $\left[1^{\prime}, 2^{\prime}: 5,6\right]$ pyrano[3,2-c] chromen-6-ones under solvent-free conditions. Bull. Chem. Soc. Ethiop. 30, 289-296 (2016)

116. Hesse, S., Kirsch, G.: A rapid access to coumarin derivatives (using Vilsmeier-Haack and Suzuki cross-coupling reactions). Tetrahedron Lett. 43, 1213-1215 (2002)

117. Lee, B.H., Clothier, M.F., Dutton, F.E., Conder, G.A., Johnson, S.S.: Anthelmintic beta-hydroxyketoamides (BKAs). Bioorg. Med. Chem. Lett. 8, 3317-3320 (1998)

118. Jung, J.-C., Jung, Y.-J., Park, O.-S.: A convenient one-pot synthesis of 4-hydroxycoumarin, 4-hydroxythiocoumarin, and 4-hydroxyquinolin-2(1H)-one. Synth. Commun. 31, 1195-1200 (2001)

119. Melagraki, G., Afantitis, A., Igglessi-Markopoulou, O., Detsi, A., Koufaki, M., Kontogiorgis, C., Hadjipavlou-Litina, D.J.: Synthesis and evaluation of the antioxidant and anti-inflammatory activity of novel coumarin-3-aminoamides and their alpha-lipoic acid adducts. Eur. J. Med. Chem. 44, 3020-3026 (2009)

120. Jung, J.-C., Lee, J.-H., Oh, S., Lee, J.-G., Park, O.-S.: Synthesis and antitumor activity of 4-hydroxycoumarin derivatives. Bioorg. Med. Chem. Lett. 14, 5527-5531 (2004)

121. Goswami, P.: Dually activated organo- and nano-cocatalyzed synthesis of coumarin derivatives. Synth. Commun. 39, 22712278 (2009)

122. Kumar, B.V., Naik, H.S.B., Girija, D., Kumar, B.V.: ZnO nanoparticle as catalyst for efficient green one-pot synthesis of coumarins through Knoevenagel condensation. J. Chem. Sci. 123, 615-621 (2011)

123. Ghosh, P.P., Das, A.R.: Nano crystalline ZnO: a competent and reusable catalyst for one pot synthesis of novel benzylamino coumarin derivatives in aqueous media. Tetrahedron Lett. 53, 3140-3143 (2012)

124. Paul, S., Bhattacharyya, P., Das, A.R.: One-pot synthesis of dihydropyrano[2,3-c]chromenes via a three component coupling of aromatic aldehydes, malononitrile, and 3-hydroxycoumarin catalyzed by nano-structured $\mathrm{ZnO}$ in water: a green protocol. Tetrahedron Lett. 52, 4636-4641 (2011)

125. Ghosh, P.P., Das, A.R.: Nanocrystalline and reusable $\mathrm{ZnO}$ catalyst for the assembly of densely functionalized $4 H$-chromenes in aqueous medium via one-pot three component reactions: a greener "NOSE" approach. J. Org. Chem. 78, 6170-6180 (2013)
126. Zaki, M.E.A., Soliman, H.A., Hiekal, O.A., Rashad, A.E.Z.: Pyrazolopyranopyrimidines as a class of anti-inflammatory agents. Naturforsch C. 61, 1-5 (2006)

127. Abdelrazek, F.M., Metz, P., Metwally, N.H., El-Mahrouky, S.F.: Synthesis and molluscicidal activity of new cinnoline and pyrano [2,3-c]pyrazole derivatives. Archiv der Pharm. 339, 456-460 (2006)

128. Tekale, S.U., Kauthale, S.S., Jadhav, K.M., Pawar, R.P.: Nano$\mathrm{ZnO}$ catalyzed green and efficient one-pot four-component synthesis of pyranopyrazoles. Sci. World J. 2013, 840954 (2013)

129. Sachdeva, H., Saroj, R.: ZnO Nanoparticles as an efficient, heterogeneous, reusable, and ecofriendly catalyst for four-component one-pot green synthesis of pyranopyrazole derivatives in water. Sci. World J. 2013, 680671 (2013)

130. Heravi, M.M., Daraie, M.: A novel and efficient five-component synthesis of pyrazole based pyrido[2,3- $d$ ] pyrimidine-diones in water: a triply green synthesis. Molecules 21, 441-452 (2016)

131. Siddiqui, Z.N., Ahmed, N., Farooq, F., Khan, K.: Highly efficient solvent-free synthesis of novel pyranyl pyridine derivatives via $\beta$-enaminones using $\mathrm{ZnO}$ nanoparticles. Tetrahedron Lett. 54, 3599-3604 (2013)

132. Haerizade, B.N., Kassaee, M.Z.: Nano ZnO promoted synthesis of 1,3-oxazoline-2-thione derivatives. J. Chem. Res. 38, 295-296 (2014)

133. Sangshetti, J.N., Dharmadhikari, P.P., Chouthe, R.S., Fatema, B., Lad, V., Karande, V., Darandale, S.N., Shinde, D.B.: Microwave assisted nano $\left(\mathrm{ZnO}-\mathrm{TiO}_{2}\right)$ catalyzed synthesis of some new 4,5,6,7-tetrahydro-6-((5-substituted-1,3,4-oxadiazol-2-yl) methyl)thieno[2,3-c]pyridine as antimicrobial agents. Bioorg. Med. Chem. Lett. 23, 2250-2253 (2013)

134. Ziraka, M., Azinfara, M., Khalilia, M.: Three-component reactions of kojic acid: efficient synthesis of dihydropyrano[3,2-b] chromenediones and aminopyranopyrans catalyzed with nano$\mathrm{Bi}_{2} \mathrm{O}_{3}-\mathrm{ZnO}$ and nano-ZnO. Curr. Chem. Lett. 6, 105-116 (2017)

135. Albadi, J., Alihosseinzadeh, A., Mansournezhad, A.: Regioselective synthesis of 1,2,3-triazoles catalyzed over $\mathrm{ZnO}$ supported copper oxide nanocatalyst as a new and efficient recyclable catalyst in water. Acta Chim. Slov. 62, 617-624 (2015)

\section{Publisher's Note}

Springer Nature remains neutral with regard to jurisdictional claims in published maps and institutional affiliations. 